\title{
Universal tangle invariant and commutants of quantum algebras
}

\author{
H C Lee \\ Physics Department, National Central University, Chungli, Taiwan 320, Republic of China $\dagger$
}

Received 25 April 1995

\begin{abstract}
We construct a universal tangle invariant on a quantum algebra. We show that the invariant maps tangle to commutants of the algebra; every $(1,1)$-tangle is mapped to a Casimir operator of the algebra; the eigenvalue of the Casimir operator in an irreducible representation of the algebra is a link polynomial for the closure of the tangle. This result is applied to a discussion of the Alexander-Conway polynomial and quantum holonomy in Chern-Simons theory in three dimensions.
\end{abstract}

\section{Introduction}

Invariants for oriented tangles valued on finite dimensional representations of quasitriangular Hopf algebras were constructed by Turaev (1990), Reshetikhin (1990) and Reshetikhin and Turaev (1990). The construction was based on the relation between categories of oriented tangles and categories of finite dimensional representations of an algebra (Yetter 1988, Freyd and Yetter 1989). A universal invariant for links valued on a quantum group was constructed by Lawrence (1989).

In this paper we construct a universal invariant for oriented tangles on a quasitriangular Hopf algebra $\mathcal{U}$ (Drinfel'd 1986, Jimbo 1985), which we call a quantum algebra. We further show that the image of (the plane projection of) a tangle on $\mathcal{U}$ is a commutant of $\mathcal{U}$ to within a permutation determined by the tangle. In particular, the image of a $(1,1)$-tangle is a central element of $\mathcal{U}$ whose eigenvalue in an irreducible representation of $\mathcal{U}$ is an invariant for the closure of the tangle. Our construction is a non-trivial extension of the result of Lawrence (1989) because a tangle lacks the crucial property possessed by a link—closure — that makes either Alexander's theorem (Alexander 1928) or Markov theorem (Markov 1935) useful. In fact, our universal invariant for links differs from that of Lawrence.

The rest of the paper is presented as follows. In section 2 we first define notation and review the main result of Lee (1992): every $(n, n)$-tangle diagram can be transformed to a partially closed braid with $n$ strands unclosed using only a special type of Reidemeister II moves. We then construct a universal tangle invariant as a functor $\mathcal{V}:[T] \rightarrow \mathcal{U}_{0}$, where $[T]$ is a tangle isotopy and $\mathcal{U}_{0}$ is an universal enveloping algebra with specific properties. In section 3 we show that one can define a homomorphism between a quantum algebra $\mathcal{U}$ and $\mathcal{U}_{0}$ that maps a central element $\lambda \in \mathcal{U}$ to the identity in $\mathcal{U}_{0}$. This requires certain refinements on the construction given in section 2 depending on whether one's objective is a universal invariant for regular isotopic tangles, ambient isotopic tangles or ribbon graphs. In section 4 we show that, to within permutations, the universal tangle invariants are commutants of $\mathcal{U}$,

† internet: hclee@halley.phy.ncu.edu.tw 
that is, they commute with the subset $\mathcal{U}_{\triangle}$ of $\mathcal{U}$ induced by the action of the coproduct on $\mathcal{U}$. This is a generalization of the coboundary condition $\mathcal{R} \Delta(\mathcal{U})=\Delta^{\prime}(\mathcal{U}) \mathcal{R}$ by replacing $\mathcal{R}$ by a universal tangle invariant and $\Delta(\mathcal{U})$ by $\mathcal{U}_{\triangle}$. In particular, $\mathcal{V}$ maps every $(1,1)$-tangle to a central element in $\mathcal{U}$. In section 5 we show that in an irreducible representation of $\mathcal{U}$ the eigenvalue of such a central element is a link polynomial for the closure of the $(1,1)$ tangle. This gives new meaning to a link polynomial as an object in a quantum algebra. In section 6 we compare our universal link invariant with that given by Lawrence (1989). In section 7 we give two simple applications of our results. In an appendix we list the universal invariants for $(1,1)$-tangles with up to six crossings.

\section{Seifert circles and a functor for tangles}

\subsection{Tangles, tangle diagrams and splices}

Definition 2.1.1. An oriented (n,n)-tangle $T$ is the disjoint union of $n$ open oriented strands and an arbitrary number of oriented closed strands embedded in a cylinder in a 3-manifold, with all the $n$ tails of the open strands held fixed on the ceiling of the cylinder and all the $n$ tips held fixed on the floor. A link is a $(0,0)$-tangle.

Remark. One could replace the cylinder with a 3-ball, and identify the region on the surface of the ball enclosing the $n$ tails (tips, resp.) as the ceiling (floor, resp.). An ( $n, m)$-tangle, $m \neq n$, amounts to a different partition of the ends of the tangle. It is necessary to include such tangles in the category theory approach (Turaev 1990, Reshetikhin 1990, Reshetikhin et al 1990, Yetter 1988, Freyd and Yetter 1989), but not in our discussion. Henceforth tangles will be understood to be $(n, n)$ and oriented.

Definition 2.1.2. A tangle diagram is a regular plane projection of a tangle. A tangle diagram is composed of positive and negative crossings and edges. In a positive (negative) crossing the undercrossing strand is clockwise (counterclockwise) to the overcrossing strand. An edge is a section of strand between two consecutive crossings.

Example. The two basic crossings in a tangle are shown in figure 1.

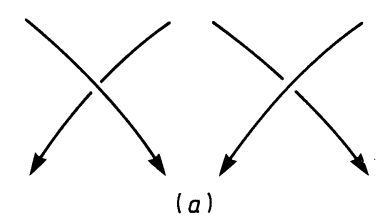

(a)

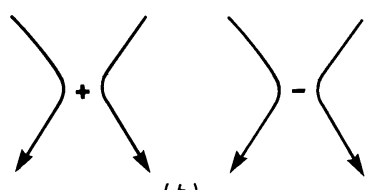

(b)

Figure 1.

Definition 2.1.3. An ambient isotopy class (simply isotopy class unless otherwise specified) of tangle diagrams, denoted by $[T]$, is an equivalence class of tangles generated by the Reidemeister moves I, II and III (figure 2) on tangle diagrams. A regular isotopy of tangles, denoted by $[T]_{\mathrm{reg}}$, is an equivalence class of tangles generated by the Reidemeister moves II and III. By a functor for tangles we mean a functor from the category whose morphisms are isotopy classes of tangle diagrams. 
Remark. Reidemeister moves are defined for diagrams with non-oriented strands. For oriented tangle diagrams it is necessary to speak of Reidemeister moves on diagrams with oriented strands.

Definition 2.1.4. If the two strands in a Reidemeister move II have the same (opposite, resp.) orientation, denote the move IIa (IIb, resp.). The two types of Reidemeister move II's are shown in figure 3.
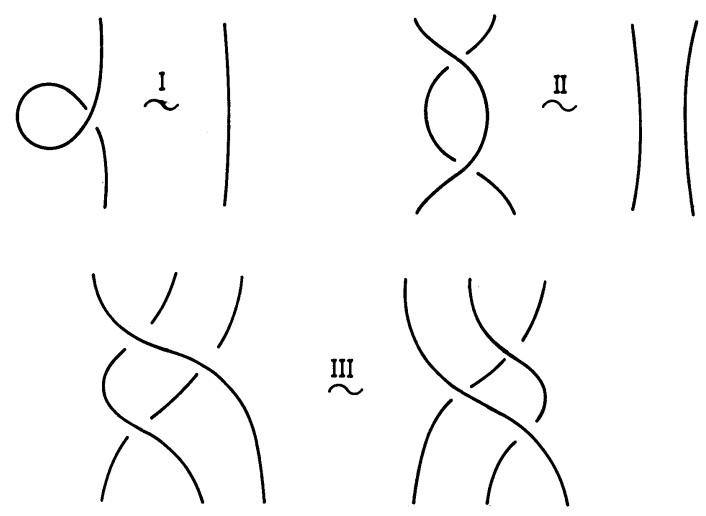

Figure 2.
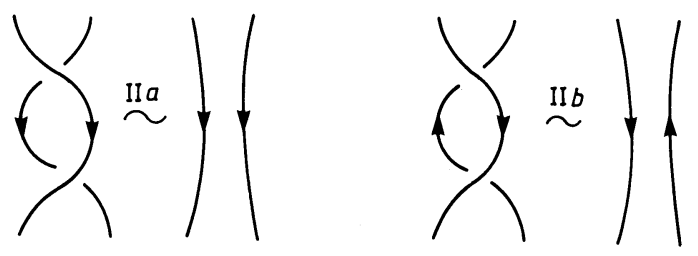

Figure 3.

Definition 2.1.5. Splicing a crossing means cutting out the vertex of the crossing, placing $\mathrm{a}+$ or - sign at the cut-out in accordance with the sign of the crossing, and reconnecting the severed strands in such a way that: (i) the orientations of the strands are respected; and (ii) the reconnected strands do not cross.

Definition 2.1.6. A splice of a tangle diagram is obtained from a tangle diagram by splicing all the crossings on the tangle.

Example. The splices of the two crossings in figure 1(a) are shown in figure 1(b).

Remark. By definition there are no crossings in a splice. Hence lines in a splice do not correspond to strands in the tangle diagram it presents. On the other hand, it is clear that the correspondence between the edges on a tangle diagram and those on its splice is one-to-one. So is the correspondence between a tangle diagram and its splice. Often, arguments used in the ensuing discussion do not depend critically on the signs at the crossing points in a 


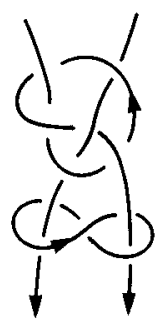

(a)

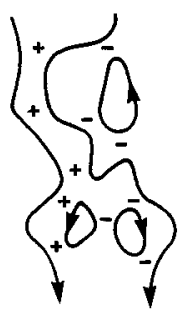

(b)

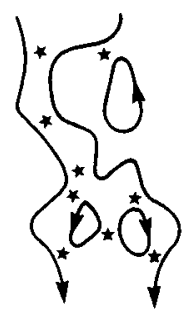

(c)

Figure 4.
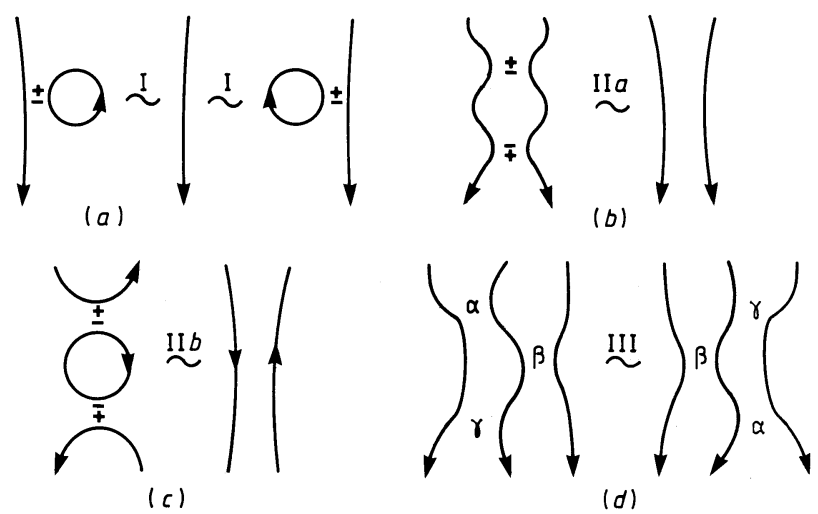

Figure 5.

splice. In such cases, the signs in the splice will be generically replaced by stars ( $\star$ 's). In figure $4,(a)$ is a tangle diagram, $(b)$ is its splice, $c$ is the splice in which the signs are replaced by $\star$ 's. The information contained in such a splice is precisely the same as that in a 4-valent planar graph.

Definition 2.1.7. The circles in a splice are called Seifert circles.

Example. There are three Seifert circles in the splice of figure 4(b) (and 4(c)).

Definition 2.1.8. The writhe and the Seifert number are, respectively, the number of positive signs minus the number of negative signs, and the number of Seifert circles, respectively, in a splice.

Example The Reidemeister moves I, IIa, IIb and III on splices are shown in figure 5(a)$(d)$, respectively. For the Reidemeister moves IIa and IIb, the two signs in the splice on the left-hand side must be opposite. Note that the left-hand side splice in IIb has a Seifert circle while that in IIa does not.

Remark. For the Reidemeister III move, in each of the two diagrams in figure $5(d)$ label the three signs, from top to bottom, respectively, by $(\alpha, \beta, \gamma)$, and use the subscripts $\mathrm{L}$ and $\mathrm{R}$, respectively, to denote the left and right diagrams. Then the precise relation is $(\alpha, \beta, \gamma)_{\mathrm{L}}=(\gamma, \beta, \alpha)_{\mathrm{R}}$, in which, of the total of eight sets of signs, the two sets 
$(\alpha, \beta, \gamma)=(+,-,+)$ and $(-,+,-)$ are excluded. It is evident that in a splice neither the writhe nor the Seifert number is an invariant of isotopy. Move I preserves neither the writhe nor the Seifert number, whereas moves IIa and III preserve both. Move IIb preserves writhe, but not necessarily the Seifert number.

Definition 2.1.9. The Reidemeister move IIb is type 2, denoted by IIb2 (type 1, denoted by IIb1, resp.) if the two lines in the right-hand side diagram in figure 5(c) belong (do not belong, respectively) to the same Seifert circle or to the same open line.

Remark If neither of the two lines belonged to a Seifert circle, then they would both belong to separate open lines in the splice, and it would have been impossible to make a Reidemeister II move on them. Thus, move IIb1 preserves the Seifert number (figure 6(a)), and move IIb2 (figure $6(b)$ ) changes the Seifert number by \pm 2 , but preserves the number of clockwise Seifert circles minus the number of counterclockwise Seifert circles.

Definition 2.1.10. Among all the edges involved in a Reidemeister IIb1 move, there is one and only one edge that belongs to a Seifert circle or to an open line both before and after the move. We speak of this edge as preserving its identity in the move.

Example. In figure 7, the identity of the edge marked with a cross is preserved.

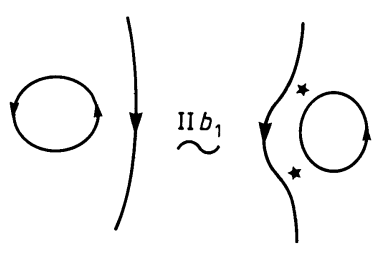

(a)

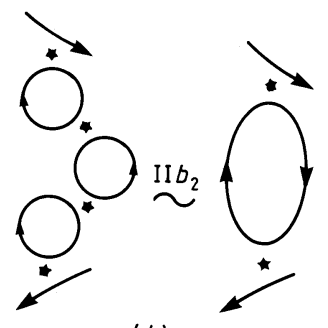

(b)

Figure 6.
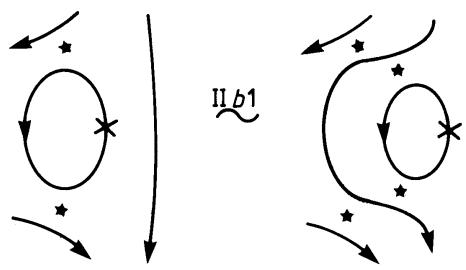

Figure 7. 


\subsection{Partially closed braids and wrong-way edges}

Definition 2.2.1. A braid is a tangle diagram whose splice does not have any Seifert circles.

Definition 2.2.2. An (n, $n)$-braid-tangle is a partially closed braid obtained by closing $m$ strands of an $(n+m)$-strand braid without disturbing any crossing in the braid.

Remark. Since the action of closing a strand in a braid must not generate any new crossing, a braid-tangle is obtained by closing the $m_{1}$ left-most strands clockwise and the $m_{2}$ rightmost strands counterclockwise, $m_{1}+m_{2}=m$. Obviously, $m+1$ possibly distinct braidtangles may be obtained.

Definition 2.2.3. The $m$ edges that close an $(n+m)$-strand braid to obtain an $(n, n)$-braidtangle are wrong-way edges of the braid- tangle. If a component of a braid-tangle that is closed contains no vertices, then the whole component is considered a wrong-way edge.

Proposition 1. Every $(n, n)$-tangle diagram can be isotopically transformed to a $(n, n)$ braid-tangle using only Reidemeister IIb1 moves.

Corollary 1.0. Every $(n, n)$-tangle diagram is isotopic to an $(n, n)$-braid-tangle.

Corollary 1.1. Every tangle diagram can be isotopically transformed to a braid-tangle such that the writhe number and the numbers of the clockwise and counterclockwise Seifert circles are separately preserved.

Corollary 1.2. For every tangle diagram there exists at least one assignment of wrongway edges, one on each Seifert circle in the splice of the tangle diagram, and a braid-tangle isotopic to the tangle diagram, such that

(i) the identities of the wrong-way edges are preserved in the transformation from the tangle diagram to the braid-tangle; and

(ii) the set of wrong-way edges on the tangle diagram coincides with the set of wrongway edges on the braid-tangle.

Proof. Proofs are given in Lee (1992).

Remark. The corollaries are fairly straightforward consequences of proposition 1 and the properties of the Reidemeister IIb1 move.

Definition 2.2.4. Given a tangle diagram $T$, a specified tangle diagram $T^{*}$ of $T$ denotes $T$ equipped with a specific choice of wrong-way edges.

\subsection{The algebra $\mathcal{U}_{0}$}

Definition 2.3.1. $\mathcal{U}_{0}$ is the tensor algebra of an algebra $\mathcal{A}$ over $\mathbb{C}$, equipped with $h, h^{\prime} \in \mathcal{A}$, $R=\sum_{i} a_{i} \otimes b_{i} \in \mathcal{A} A, R^{\prime}=\sum_{i} b_{i}^{\prime} \otimes a_{i}^{\prime} \in \mathcal{A} A$ satisfying

$$
\begin{aligned}
& h h^{\prime}=h^{\prime} h=1 \\
& R\left(\mathcal{T} R^{\prime}\right)=R^{\prime}(\mathcal{T} R)=1 \otimes 1 \\
& R_{12} R_{13} R_{23}=R_{23} R_{13} R_{12} \\
& A h B=B^{\prime} h A^{\prime}=B h^{\prime} A=A^{\prime} h^{\prime} B^{\prime}=1
\end{aligned}
$$


where $A \cdots B$ is the shorthand for $\sum_{i} a_{i} \cdots b_{i}$; similarly for $B^{\prime} \cdots A^{\prime} ; \mathcal{T}(a \otimes b)=$ $b \otimes a ; R_{12}=R \otimes 1 ; R_{13}=A \otimes 1 \otimes B ; R_{23}=1 \otimes \mathcal{R}$. The multiplication $m$ in $\mathcal{U}_{0}$ is a morphism, $m: \mathcal{A} \rightarrow \mathcal{A}$, by $a \otimes b \mapsto m(a \otimes b) \equiv a b, a, b \in \mathcal{A}$. By (2.2) we define $R^{-1}=\mathcal{T} R^{\prime}, R^{\prime-1}=\mathcal{T} R$.

Definition 2.3.2. Consider $\mathcal{A}$ as a linear vector space. Let $\mathcal{I} \subset \mathcal{A}$ be spanned by $a b-b a, \forall a, b \in \mathcal{A}, \mathcal{A}_{0} \equiv \mathcal{A} / \mathcal{I}$ and the natural projection $S p: \mathcal{A} \rightarrow \mathcal{A}_{0}$. Then

$$
\operatorname{Sp}(a b)=\operatorname{Sp}(b a) \quad \forall a, b \in \mathcal{A} .
$$

In what follows we consider $S p$ as part of the structure of $\mathcal{U}_{0}$.

\subsection{The functor $\mathcal{V}$ for tangles}

Let $\{T\}$ denote the set of all tangles; [T] denote an isotopy class of tangles and $T_{l}^{(n)}$ denote an $(n, n)$-tangle diagram with $l$ closed strands.

Definition 2.4.1. Consider the splice of a specified tangle diagram $T^{*}$. Call a wrong-way edge on a counterclockwise Seifert circle an A-edge (diagrammatically represented by $\uparrow$ or, equivalently, by $\downarrow$ ), and one on a clockwise circle a $C$-edge $(\uparrow$ or $\downarrow)$. An edge that is neither an A-edge nor a C-edge is an $N$-edge ( $\uparrow$ or $\downarrow)$. The property of an edge does not depend on its relative direction in the tangle. We refer to all these edges as arrows. Apply these definitions also to $T^{*}$.

Definition 2.4.2. Let $\alpha, \beta, \ldots$ be a set of arrows. The tensor product $\alpha \otimes \beta$ is the disjoint union of the two arrows. The multiplication $p$ acting on $\alpha \otimes \beta$ adjoins the head of $\alpha$ to the tail of $\beta: p(\alpha \otimes \beta) \equiv p_{12}(\alpha \otimes \beta)=\alpha \beta$. Also $p_{13}(\alpha \otimes \beta \otimes \tau)=\alpha \tau \otimes \beta, p_{31}(\alpha \otimes \beta \otimes \tau)=\tau \alpha \otimes \beta$. Successive multiplications give an open string of arrows (henceforth simply a string). The notions of multiplying and tensoring arrows extend to strings.

Definition 2.4.3. Given a string $\alpha_{1} \alpha_{2} \cdots \alpha_{k}$, let $\hat{\tau}_{\{\alpha\}}$ be the class $\left\{\alpha_{s_{1}} \alpha_{s_{2}} \cdots \alpha_{s_{k}} \mid\left(s_{1}, s_{2}, \ldots\right.\right.$, $\left.s_{k}\right) \in\{$ all cyclic perm. of $\left.(1,2, \ldots, k)\}\right\}$ and refer to it as a closed string of arrows, or simply a closed string.

Remark. Thus a specified tangle diagram $T^{*}$ is a disjoint union of open and closed strings composed of the arrows $\downarrow, \downarrow$ and $\downarrow$, and pairs of tensored arrows $\downarrow$ (overcrossing) and $\downarrow$ (undercrossing), equipped with the operations multiplication and tensor product.

Definition 2.4.4. Let $h, h^{\prime}, R, R^{\prime}$ and $S p$ be the objects in $\mathcal{U}_{0}$ defined in section 2.3. Let $\alpha, \beta$ be strings, $\hat{\tau}$ be a closed string in $T^{*}$ and $\left[T^{*}\right]$ be the set of all specified tangle diagrams. Define the map $\mathcal{V}:\left[T^{*}\right] \rightarrow \mathcal{U}_{0}$ by

$$
\begin{aligned}
& \mathcal{V}(\downarrow)=\mathcal{V}(\uparrow)=1 \quad \mathcal{V}(\gg)=\mathcal{V}(\downarrow)=h \quad \mathcal{V}(\uparrow)=\mathcal{V}(\downarrow)=h^{\prime} \\
& \mathcal{V}(\searrow)=R \quad \mathcal{V}\left(\swarrow_{\downarrow}\right)=R^{\prime} \\
& \mathcal{V}(\alpha \otimes \beta)=\mathcal{V}(\alpha) \otimes \mathcal{V}(\beta) \\
& \mathcal{V}(\alpha \beta)=m(\mathcal{V}(\alpha) \otimes \mathcal{V}(\beta))=\mathcal{V}(\alpha) \mathcal{V}(\beta) \\
& \mathcal{V}(\hat{\tau})=\operatorname{Sp}\left(\mathcal{V}\left(\tau_{*}\right)\right) \in \mathcal{A}_{0} \quad \tau_{*} \in \hat{\tau} .
\end{aligned}
$$

Proposition 2. $\mathcal{V}$ is a functor for tangles; it is well defined as a map $\mathcal{V}:\{T\} /$ isotopy $\rightarrow$ $\mathcal{U}_{0},\left[T_{l}^{(n)}\right] \mapsto \mathcal{V}\left[T_{l}^{(n)}\right]=\mathcal{V}\left(T^{*}\right) \in \mathcal{A} n n \otimes \mathcal{A}_{0}^{\otimes l}, \forall T \in\left[T_{l}^{(n)}\right]$. 
Proof. The proof is given in Lee (1992). There, it is shown that if (i) $T_{a}$ and $T_{b}$ are isotopic; (ii) $T_{a}^{*}$ is a specified tangle diagram of $T_{a}$ and (iii) $T_{b}^{*}$ is a specified tangle diagram of $T_{b}$ then $\mathcal{V}\left(T_{a}^{*}\right)=\mathcal{V}\left(T_{b}^{*}\right)$.

Remark. $\mathcal{V}$ maps each open string in $T^{*}$ to a factor valued in $\mathcal{A}$ and each closed string in $T^{*}$ to a factor valued in $\mathcal{A}_{0}$. The cyclicity of $S p$ in (2.5) is needed for (2.10).

Example. The specified tangle diagram $T^{*}$ in figure $8(a)$, a counterclockwise writhe with an overcrossing, may be expressed as $T^{*}=p_{12}\left(p_{13}(\chi \otimes \uparrow)\right)$. From the rules given above, $\mathcal{V}\left(T^{*}\right)=m_{12}\left(m_{13}(R \otimes h)\right)=m_{12}(A h \otimes B)=A h B ;$ see figure $8(b)$.

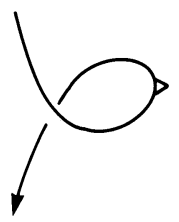

(a)

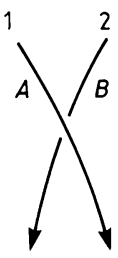

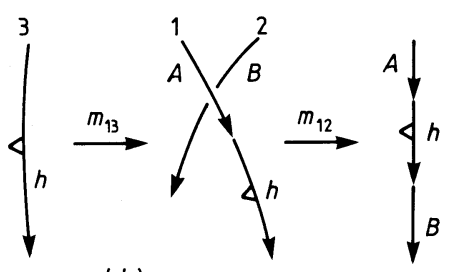

(b)

Figure 8.

\subsection{Some properties of $\mathcal{V}$}

2.5.1. Definition 2.5.1. Define an equivalence relation $\sim$ on tangle diagrams by saying that $U \sim W$ if, and only if, $\mathcal{V}\left(U^{*}\right)=\mathcal{V}\left(W^{*}\right)$ for all specified tangle diagrams $U^{*}$ and $W^{*}$ whose tangle diagrams are $U$ and $W$, respectively.

Example. The basic relations (2.2-4) of the algebra $\mathcal{U}_{0}$ are shown diagrammatically in figure $9(a)-(c)$.

Remark. Equation (2.4) is the algebraic expression of the Reidemeister I move (figure 9(c)). Hence, unless the elements $h$ and $h^{\prime}$ are equal to the identity, it is necessary to differentiate wrong-way edges from other edges.

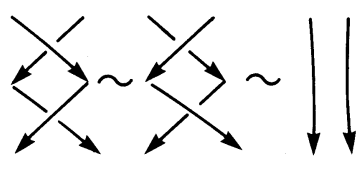

(a)

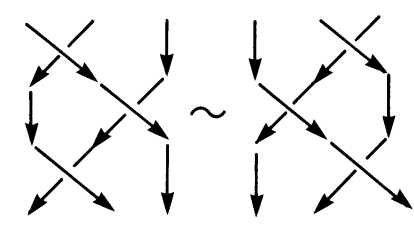

(b)

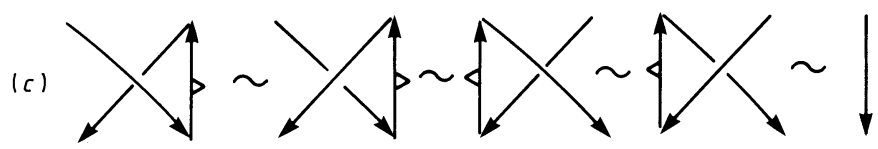

Figure 9. 
2.5.2. Following are some derived Lee (1992) relations shown diagrammatically in figures $10-12$, respectively.

$A^{\prime} A \otimes B h^{\prime} B^{\prime}=B B^{\prime} \otimes A^{\prime} h^{\prime} A=1 \otimes h^{\prime} \quad B^{\prime} h B \otimes A A^{\prime}=A h A^{\prime} \otimes B^{\prime} B=h \otimes 1$

$R(h \otimes h) R^{-1}=R^{\prime}(h \otimes h) R^{\prime-1}=h \otimes h, R\left(h^{\prime} \otimes h^{\prime}\right) R^{-1}=R^{\prime}\left(h^{\prime} \otimes h^{\prime}\right) R^{\prime-1}=h^{\prime} \otimes h^{\prime}$

$[h \otimes h, R]=\left[h \otimes h, R^{\prime}\right]=\left[h^{\prime} \otimes h^{\prime}, R\right]=\left[h^{\prime} \otimes h^{\prime}, R^{\prime}\right]=0$.
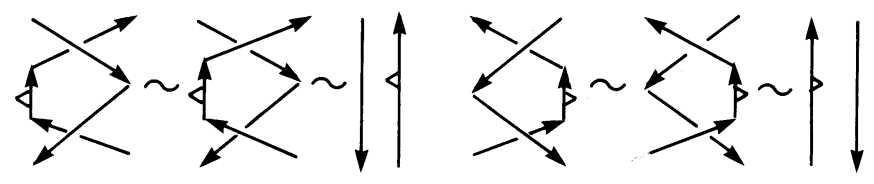

Figure 10.
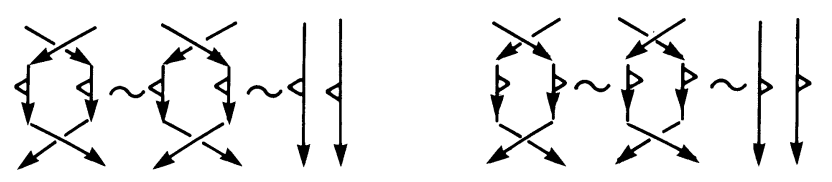

Figure 11.
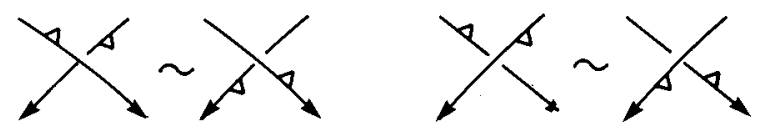

Figure 12.

Remark. Equation (2.11) is the algebraic expression of the Reidemeister IIb move (figure 10). A graphical derivation of the second relation in (2.12) is given in figure 13.

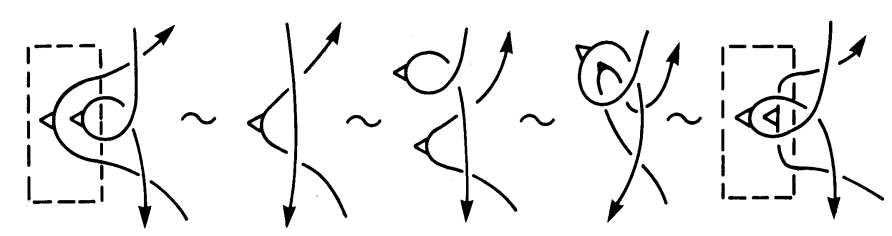

Figure 13.

2.5.3. If $\mathcal{B}$ is a braid of $n$ strands, then repeated application of (2.13) gives

$$
\left[h^{\otimes n}, \mathcal{V}(\mathcal{B})\right]=\left[h^{\prime \otimes n}, \mathcal{V}(\mathcal{B})\right]=0
$$


2.5.4. Definition 2.5.2. For the set of $(n, n)$-tangles $\left\{T^{(n)}\right\}$, let $\sigma:\left\{T^{(n)}\right\} \rightarrow S_{n}$ be the natural mapping of tangles to the permutation group. We say $\sigma \in S_{n}$ sends $\{1,2, \ldots, n\}$ to $\left\{i_{1}, i_{2}, \ldots, i_{n}\right\}$ when $\sigma\left(a_{1} \otimes a_{2} \otimes \cdots \otimes a_{n}\right)=a_{i_{1}} \otimes a_{i_{2}} \otimes \cdots \otimes a_{i_{n}}$.

Let $T_{a}$ and $T_{b}$ be two $(n, n)$-tangles and let $T_{a b}$ be obtained by joining in order the tips of $T_{a}$ to the tails of $T_{b}$, then

$$
\mathcal{V}\left[T_{a b}\right]=\mathcal{V}\left[T_{a}\right] \sigma\left(T_{a}\right)\left(\mathcal{V}\left[T_{b}\right]\right)
$$

2.5.5. Let $T$ be an $(n, n)$-tangle with $l$ closed strands. There exists a braid $\mathcal{B}$ of $N=n+\mu_{\mathrm{L}}+\mu_{\mathrm{R}}$ strands, and a braid-tangle $T_{\mathcal{B}}$, obtained from $\mathcal{B}$ by closing the latter's $\mu_{\mathrm{L}}$ left-most strands clockwise and $\mu_{\mathrm{R}}$ right-most strands counterclockwise, such that $\left(T_{\mathcal{B}}\right)$ may be reached by executing only Reidemeister IIb1 moves on $T$. Suppose $\sigma(\mathcal{B})$ sends $\{1,2, \ldots, N\}$ to $\left\{i_{1}, i_{2}, \ldots, i_{N}\right\}$. Let $\beta=\mathcal{V}(\mathcal{B}), \mathcal{H}=h^{\prime \otimes \mu_{\mathrm{L}}} \otimes 1^{\otimes n} \otimes h^{\otimes \mu_{\mathrm{R}}}$, and $m_{\left\{T_{\mathcal{B}}\right\}}$ be an $\left(\mu_{\mathrm{L}}+\mu_{\mathrm{R}}\right)$-fold composition of multiplications:

$$
m_{\left\{T_{\mathcal{B}}\right\}}=\left(\prod_{s=1}^{\mu_{\mathrm{L}}}\left(\circ m_{i_{s}, s}\right)\right) \circ 1^{\otimes n}\left(\prod_{s=N-\mu_{\mathrm{R}}+1}^{N}\left(\circ m_{i_{s}, s}\right)\right) .
$$

This defines the action that closes $\mathcal{B}$ to yield $T_{\mathcal{B}}$. Because there are $l$ closed strands in $T$, there must be $l$ cycles among the set of pairs of subscripts $\left\{i_{s}, s\right\}$ in (2.16). (For example, a cycle involving three pairs is a set $\left(\left\{s_{1}, s_{2}\right\},\left\{s_{2}, s_{3}\right\},\left\{s_{3}, s_{1}\right\}\right)$, with $s_{1}, s_{2}$ and $s_{3}$ different.) We also have $l \leqslant \mu_{\mathrm{L}}+\mu_{\mathrm{R}}$ and $\sigma\left(T_{\mathcal{B}}\right) \in S_{n}$ sending $j$ to $\sigma(\mathcal{B})\left(\mu_{\mathrm{L}}+j\right)-\mu_{\mathrm{L}}=i_{\mu_{\mathrm{L}}+j}-\mu_{\mathrm{L}}$.

2.5.6. Denote by $S p^{\otimes l}$ the action that sends the $l$ closed strands to $\mathcal{A}_{0}^{\otimes l}$ according to definition 2.4.3 and (2.10), and define $C l\left\{T_{\mathcal{B}}\right\}=S p^{\otimes l} \circ m_{\left\{T_{\mathcal{B}}\right\}}$. If [T] is the isotopy of $T_{\mathcal{B}}^{*}$, then the map $\mathcal{V}$ for $[T]$ is explicitly given in terms of $C l\left\{T_{\mathcal{B}}\right\}, \sigma(\mathcal{B})$ and $\mathcal{H}$ by

$$
\mathcal{V}[T]=\mathcal{V}\left(T_{\mathcal{B}}^{*}\right)=C l\left\{T_{\mathcal{B}}\right\}(\mathcal{H} \beta)=C l\left\{T_{\mathcal{B}}\right\}(\beta \sigma(\mathcal{B})(\mathcal{H})) .
$$

The following are two simple examples. Suppose $T_{\mathrm{R}}$ is obtained from $T$ by closing the latter's right-most strand counterclockwise, and $\sigma(T)$ sends $\{1,2, \ldots, n\}$ to $\left\{i_{1}, i_{2}, \ldots, i_{n}\right\}$. Then

$$
\mathcal{V}\left[T_{\mathrm{R}}\right]=S p^{\otimes l_{\mathrm{R}}} \circ m_{i_{n}, n}(\mathcal{V}[T] \sigma(T)(1 \otimes \cdots \otimes 1 \otimes h))
$$

where $l_{\mathrm{R}}=1(0)$ if the closing generates (does not generate) a new closed strand in $T_{\mathrm{R}}$. Similarly, if $T_{\mathrm{L}}$ is obtained from $T$ by closing its left-most strand clockwise, then

$$
\mathcal{V}\left[T_{\mathrm{L}}\right]=S p^{\otimes L_{\mathrm{L}}} \circ m_{i_{1}, 1}\left(\mathcal{V}[T] \sigma(T)\left(h^{\prime} \otimes 1 \otimes \cdots \otimes 1\right)\right) .
$$

2.5.7. There are $n+1$ ways of closing all the strands in $T$ : closing the $m$ left-most strands clockwise on the left, and the rest of the strands counterclockwise on the right, $m=0,1, \ldots, n$. Refer to the links thus obtained by $\hat{T}_{m}$. Clearly all $\hat{T}_{m}$ 's belong to the same class, $[\hat{T}]$, the generic closure of $T$. Define

$$
\mathcal{H}_{m} \equiv h^{\otimes m} \otimes h^{\prime \otimes(n-m)} \in \mathcal{A} n n \quad m=0,1, \ldots, n .
$$

Then $\hat{T}_{m}$ being isotopic to $[\hat{T}]$ demands that the following equality holds:

$$
\mathcal{V}[\hat{T}]=\mathcal{V}\left[\hat{T}_{m}\right]=C l\{T\}\left(\mathcal{V}[T] \sigma(T)\left(\mathcal{H}_{m}\right)\right) \quad m=0,1, \ldots, n .
$$

Since $T$ itself is the partial closure of a braid, it is sufficient to prove the equality when $T$ is a braid $\mathcal{B}$ of $n$ strands, namely,

$$
\mathcal{V}[\hat{\mathcal{B}}]=\operatorname{Cl}\{\mathcal{B}\}\left(\mathcal{V}(\mathcal{B}) \sigma(\mathcal{B})\left(\mathcal{H}_{m}\right)\right) \quad m=0,1, \ldots, n .
$$

This relation is proved in Lee (1992).

Example. Figure 14 gives a graphical derivation of (2.21) for the case $n=3, m=2$. 


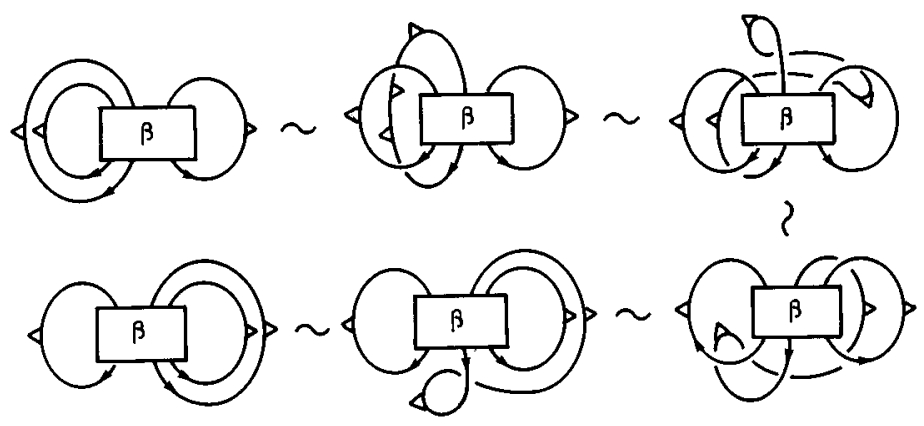

Figure 14.

\section{Functor for tangles on a quantum algebra}

\subsection{Quasitriangular Hopf algebra}

Definition 3.0.1. A quasitriangular Hopf algebra (Drinfel'd 1986) is a (not necessarily commutative or cocommutative) Hopf algebra $\{\mathcal{A}, S, m, \Delta, \epsilon\}$ equipped with an invertible element $\mathcal{R} \in \mathcal{A} \otimes \mathcal{A}$ satisfying:

$$
\begin{aligned}
& m(\mathrm{id} \otimes S) \triangle(a)=m(S \otimes \mathrm{id}) \triangle(a)=\epsilon(a) \mathbf{1} \quad \forall a \in \mathcal{A} \\
& \mathcal{R}^{-1}=(S \otimes \mathrm{id}) \mathcal{R}=\left(\mathrm{id} \otimes S^{-1}\right) \mathcal{R} \\
& \left(\triangle^{\prime}(a)\right) \mathcal{R}=\mathcal{R} \triangle(a) \quad \forall a \in \mathcal{A} \\
& (\triangle \otimes \mathrm{id}) \mathcal{R}=\mathcal{R}_{13} \mathcal{R}_{23} \quad(\mathrm{id} \otimes \triangle) \mathcal{R}=\mathcal{R}_{13} \mathcal{R}_{12} \\
& \mathcal{R}_{12} \mathcal{R}_{13} \mathcal{R}_{23}=\mathcal{R}_{23} \mathcal{R}_{13} \mathcal{R}_{12} .
\end{aligned}
$$

The antipode $S: \mathcal{A} \rightarrow \mathcal{A}$ is an antiautomorphism, and the multiplication $m: \mathcal{A} \otimes \mathcal{A} \rightarrow \mathcal{A}$, the comultiplication $\triangle: \mathcal{A} \rightarrow \mathcal{A} \otimes \mathcal{A}$ and the counit $\epsilon: \mathcal{A} \rightarrow \mathbb{C}$ are morphisms. $\triangle^{\prime}$ is the opposite comultiplication. We denote the algebra, which we also call a quantum algebra, by $\mathcal{U} ; \mathcal{U} \supset \mathcal{A}, \mathcal{A} \otimes \mathcal{A}, \mathcal{A} \otimes \mathcal{A} \otimes \mathcal{A}, \ldots$

Example. $\mathcal{U}$ may be a deformed Lie algebra (Drinfel'd 1986, Jimbo 1985, Reshetikhin et al 1990) or an algebra of functions on a quantum formal group (Faddeev 1988). An explicit example of the former is a quantized universal enveloping algebra $\mathcal{U}_{q}(g)$ over $\mathbb{C} \llbracket \eta \rrbracket, q \equiv e^{\eta}$ an indeterminate, where $g$ is a simple complex Lie algebra of rank $r$ with simple roots $\alpha_{i}, i=1, \ldots, r$ and Cartan matrix $A_{i j}=2\left\langle\alpha_{i}, \alpha_{j}\right\rangle /\left\langle\alpha_{i}, \alpha_{i}\right\rangle$, where $\langle$,$\rangle is an$ invariant scalar product. Its generators in the Chevalley basis $\left\{H_{i}, X_{i}^{+}, X_{i}^{-} ; i=1, \ldots, r\right\}$ are constrained by

$$
\begin{aligned}
& {\left[H_{i}, X_{j}^{ \pm}\right]= \pm\left\langle\alpha_{i}, \alpha_{j}\right\rangle X_{j}^{ \pm} \quad\left[X_{i}^{+}, X_{j}^{-}\right]=\delta_{i j} \frac{q^{H_{i}}-q^{-H_{i}}}{q-q^{-1}}} \\
& \sum_{\nu=0}^{m_{i j}}\left(\begin{array}{c}
m_{i j} \\
v
\end{array}\right)_{q_{i}}(-1)^{v} q_{i}^{-v\left(m_{i j}-v\right) / 2} X_{i}^{ \pm v} X_{j}^{ \pm} X_{i}^{ \pm m_{i j}-v}=0 \quad|i-j|=1 \\
& \triangle H_{i}=H_{i} \otimes 1+1 \otimes H_{i} \quad \Delta X_{i}^{ \pm}=X_{i}^{ \pm} \otimes q^{H_{i} / 2}+q^{-H_{i} / 2} \otimes X_{i}^{ \pm} \\
& \epsilon\left(H_{i}\right)=\epsilon\left(X_{i}\right)=0
\end{aligned}
$$


where $m_{i j}=1-A_{i j}, q_{i}=q^{\left\langle\alpha_{i}, \alpha_{i}\right\rangle},[a, b]=a b-b a$, and $\left(\begin{array}{c}m \\ n\end{array}\right)_{x}=\prod_{\nu=1}^{n}\left(1-x^{m-v+1}\right) /\left(1-x^{\nu}\right)$. The action of the antipode $S$ is determined by $\epsilon, \triangle$ and (3.1a):

$$
S\left(H_{i}\right)=-H_{i} \quad S\left(X_{i}^{ \pm}\right)=-q^{H_{i} / 2} X_{i}^{ \pm} q^{-H_{i} / 2} .
$$

Drinfel'd $(1985,1986)$ has shown that the $\mathcal{R}$-matrix may be expressed as an infinite formal sum in $\mathcal{A} \otimes \mathcal{A}$, defined by giving $\mathbb{C} \llbracket \eta \rrbracket$ an $\eta$-adic topology.

Remark. Often the antipode on $X_{i}$ is given a form equivalent to (3.3),

$$
S\left(X_{i}^{ \pm}\right)=-q^{\rho} X_{i}^{ \pm} q^{-\rho}
$$

where $\rho=\frac{1}{2} \sum_{\alpha \in \Delta_{+}} H_{\alpha}$, and $\Delta_{+}$is the set of positive roots of $g$.

Definition 3.0.2. Henceforth, by $\mathcal{U}$ we mean a $\mathcal{U}_{q}(g)$ such as the one given in the example above. Also denote the opposite multiplication by $m^{\prime}$ and define $\mathcal{R}^{\prime} \equiv \mathcal{T} \mathcal{R}^{-1}$.

Lemma 3.0.1. The two elements $v_{0} \equiv m((\mathrm{id} \otimes S) \mathcal{R})$ and $v_{0}^{\prime} \equiv S\left(v_{0}\right)=m^{\prime}((\mathrm{id} \otimes S) \mathcal{R})$ are invertible and satisfy, $\forall a \in \mathcal{A}$,

$$
\begin{array}{lc}
v_{0}^{-1}=m\left(\left(S^{2} \otimes \mathrm{id}\right) \mathcal{R}\right)=m\left(\left(\mathrm{id} \otimes S^{-2}\right) \mathcal{R}\right) & v_{0} a v_{0}^{-1}=S^{-2}(a) \\
v_{0}^{\prime-1}=m^{\prime}\left(\left(S^{2} \otimes \mathrm{id}\right) \mathcal{R}\right)=m^{\prime}\left(\left(\mathrm{id} \otimes S^{-2}\right) \mathcal{R}\right) & v_{0}^{\prime} a v_{0}^{\prime-1}=S^{2}(a) .
\end{array}
$$

Lemma 3.0.2. $\lambda \equiv v_{0} v_{0}^{\prime}=v_{0}^{\prime} v_{0}$ is a central element of $\mathcal{A}$ :

$$
[a, \lambda]=0 \quad \forall a \in \mathcal{A} .
$$

Proofs. Proven in Drinfel'd (1989). For completeness we give a proof below.

Notation. Throughout the rest of the paper we write $\mathcal{R}=\sum_{r} a_{r} \otimes b_{r} \equiv A \otimes B, \bar{A} \equiv S(A)$, $\underline{A} \equiv S^{-1}(A)$ so that $\mathcal{R}^{-1}=\mathcal{T} \mathcal{R}^{\prime}=\bar{A} \otimes B=A \otimes \underline{B}$. For the product of two $\mathcal{R}$ 's we write $\overline{\mathcal{R}} \mathcal{R}=A A^{\prime} \otimes B B^{\prime}$. (i.e. $A^{\prime} \otimes B^{\prime}$ stands for $\mathcal{R}$, not $\mathcal{R}^{\prime}$.)

For $a \in \mathcal{A}$ write $\triangle(a)=\sum y_{r} \otimes z_{r} \equiv Y \otimes Z$. From (3.1a) and (3.1c),

$$
0=m(\operatorname{id} \otimes S)\left(\mathcal{R}^{-1}\left(\triangle^{\prime}(a)\right) \mathcal{R}-\triangle(a)\right)=A Z v_{0} S(Y) B-Y S(Z) .
$$

Left multiply by $A^{\prime}$ and right multiply by $\bar{B}^{\prime}$ and noting that the second term on the last expression is equal to $\epsilon(a) \mathbf{1}$ and that $A^{\prime} A \otimes B \bar{B}^{\prime}=(\mathrm{id} \otimes S)\left(\mathcal{R} \mathcal{R}^{-1}\right)=\mathbf{1} \otimes \mathbf{1}$, we have

$$
Z v_{0} S(Y)=Y S(Z) v_{0}
$$

Letting $a=H_{i}$ yields $\left[v_{0}, H_{i}\right]=0$. Letting $a=X_{i}^{ \pm}$further yields $v_{0} S\left(X_{i}^{ \pm}\right)=S^{-1}\left(X_{i}^{ \pm}\right) v_{0}$. This implies $v_{0} S(a)=S^{-1}(a) v_{0}, \forall a \in \mathcal{A}$. Applying this last relation to $m\left((\operatorname{id} \otimes S) \mathcal{R}^{-1} \mathcal{R}\right)=$ $A v_{0} B=A S^{-2}(B) v_{0}=\mathbf{1}$ then identifies $A S^{-2}(B)$ as the left-inverse of $v_{0}$. This proves the second relation in (3.5). The proof of the rest of (3.5) and (3.6) is similar, which we omit. Relation (3.7) then follows directly from the last parts of (3.5) and (3.6). 
Lemma 3.0.3. $v_{0}, v_{0}^{\prime}$ satisfy

$m\left(\mathcal{R}\left(v_{0} \otimes 1\right)\right)=m^{\prime}\left(\mathcal{R}\left(v_{0}^{\prime} \otimes 1\right)\right)=1 \quad m\left(\mathcal{R}^{\prime}\left(v_{0} \otimes 1\right)\right)=m\left(\mathcal{R}^{-1}\left(v_{0}^{\prime} \otimes 1\right)\right)=\lambda$.

Proof. We prove explicitly the first and third relations and omit the proof of others:

$$
\begin{aligned}
& 1=m\left((\mathrm{id} \otimes S) \mathcal{R}^{-1} \mathcal{R}\right)=m\left((\mathrm{id} \otimes S) A A^{\prime} \otimes \underline{B} B^{\prime}\right)=A A^{\prime} \bar{B}^{\prime} B=m\left(\mathcal{R}\left(v_{0} \otimes 1\right)\right) \\
& m\left(\mathcal{R}^{\prime}\left(v_{0} \otimes 1\right)\right)=B v_{0} \bar{A}=v_{0} S^{2}(B) \bar{A}=v_{0} v_{0}^{\prime} .
\end{aligned}
$$

Remark. Recall that $\mathcal{R}$ is unchanged under the action of $(S \otimes S)$.

Definition 3.0.3. Define $v \equiv \lambda^{-1 / 2} v_{0}, v^{\prime} \equiv \lambda^{-1 / 2} v_{0}^{\prime}$.

Remark. Although there may not be an explicit expression for $\lambda^{-1 / 2}$ (or $\lambda^{1 / 2}$ ) in terms of $H_{i}$ and $X_{i}^{ \pm}$, its $\eta$-adic evaluation in $\mathbb{C} \llbracket \eta \rrbracket$ is well defined.

By definition and from (3.8), $v$ and $v^{\prime}$ satisfy

$$
\begin{aligned}
& v v^{\prime}=1 \\
& A v B=B v^{\prime} A=\lambda^{-1 / 2} \quad B v \bar{A}=\bar{A} v^{\prime} B=\lambda^{1 / 2} .
\end{aligned}
$$

Example. Relations (3.10) are illustrated in figure 15.
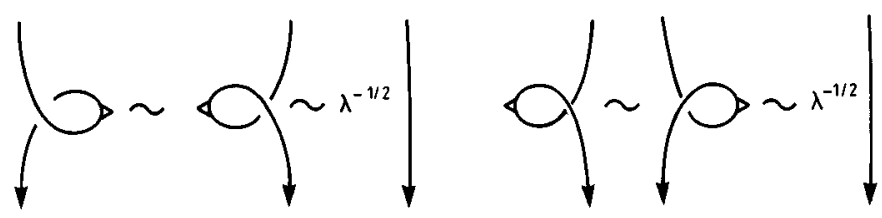

Figure 15.

\subsection{Universal invariants for tangles}

Definition 3.1.1. Let $\mathcal{V}$ be the functor of section 2.4, with the the algebra $\mathcal{U}_{0}$ of section 2.3 replaced by the quantum algebra $\mathcal{U}$, and the set $\left\{R, R^{\prime}, h, h^{\prime}\right\}$ in $\mathcal{U}_{0}$ replaced by the set $\left\{\mathcal{R}, \mathcal{T}^{-1}, v, v^{-1}\right\}$ in $\mathcal{U}$.

Proposition 3. $\mathcal{V}[T]_{\text {reg }}$ valued on $\mathcal{U}$ is a universal invariant for regular tangle isotopy.

Proof. Comparing (3.9), (3.1b), (3.1e) and (3.10) with (2.1), (2.2), (2.3) and (2.4), we can define a homomorphism between $\mathcal{U}$ and the algebra $\mathcal{U}_{0}$ of section 2.3 that maps $\left\{\mathcal{R}, \mathcal{T} \mathcal{R}^{-1}, v, v^{-1}, \lambda\right\}$ in $\mathcal{U}$ to $\left\{R, R^{\prime}, h, h^{\prime}, 1\right\}$ in $\mathcal{U}_{0}$. Note that the central element $\lambda$ in $\mathcal{U}$ is not the identity element. Since (2.4) gives the Reidemeister move I (figure $9(c)$ ), its replacement by (3.10) means that $\mathcal{V}$ does not observe the invariance of Reidemeister move I. Hence the relations (2.11) and (2.12), which establishes the equivalence relation of Reidemeister move IIb on specified tangle diagrams and were proven in Lee (1992) graphically for $\mathcal{U}_{0}$ using Reidemeister I moves, need to be re-derived without using such moves. This is done as follows. For the first relation in (2.11),

$\bar{A} A^{\prime} \otimes B^{\prime} v^{-1} B=\bar{A} A^{\prime} \otimes B^{\prime} S^{2}(B) v^{-1}=\left((\mathrm{id} \otimes S)\left(\mathcal{R} \mathcal{R}^{-1}\right)\right)\left(1 \otimes v^{-1}\right)=\left(1 \otimes v^{-1}\right)$.

For the first relation in (2.12),

$A v \bar{A}^{\prime} \otimes B v B^{\prime}=v S^{2}(A) \bar{A}^{\prime} \otimes v S^{2}(B) B^{\prime}=(v \otimes v)\left(\mathcal{R} \mathcal{R}^{-1}\right)=v \otimes v$.

The other relations are similarly derived. Proposition 3 is now obtained by replacing all reference to (ambient) isotopy in proposition 2 by regular isotopy. 
Remark. If two tangles $T$ and $T^{\prime}$ are related by Reidemeister I moves, then $\mathcal{V}\left(T^{*}\right)$ and $\mathcal{V}\left(T^{\prime *}\right)$ may differ by factors of $\lambda^{ \pm 1 / 2}$.

Definition 3.1.2. Let $w_{i}$ be the total number of positive crossings minus the total number of negative crossings on the $i$ th component in an $l$-component tangle diagram $T$. The writhe number of the tangle is $w(T)=\sum_{i=1}^{l} w_{i} / 2$. Define the functor $\mathcal{V}_{A}$ acting on $T$ by

$$
\mathcal{V}_{A}(T)=\left(\otimes_{i=1}^{l} \lambda^{w_{i} / 4}\right) \mathcal{V}\left(T^{*}\right) .
$$

Corollary 3.1. The functor $\mathcal{V}_{A}:\{T\} \rightarrow \mathcal{U}$ by $\mathcal{V}_{A}[T]=\mathcal{V}_{A}(T)$ is an invariant for ambient isotopic tangles.

Proof. $\mathcal{V}\left(T^{*}\right)$ gives a regular isotopy. The writhe number is preserved in Reidemeister II and III moves. Every Reidemeister I move on the $i$ th component induces a $\lambda^{\mp 1 / 2}$ factor in the functor and changes $w_{i}$ by $\pm 1 / 2$. Hence the factor $\left(\otimes_{i} \lambda^{w_{i} / 4}\right)$ in (3.13).

\subsection{Invariants for ribbon graphs}

Definition 3.2.1. A ribbon graph (Reshetikhin and Turaev 1990) is a tangle diagram with the strands replaced by ribbons that admit twists. A ribbon graph without any twists is a flat ribbon graph. A full clockwise (counterclockwise) twist on a ribbon is equivalent to a writhe on a flat ribbon with positive (negative) crossing. See figure 16. There is a natural one-to-one correspondence between a flat ribbon graph and a tangle diagram. Given a ribbon graph $G$, denote by $T_{G}$ the tangle diagram corresponding to the flat ribbon graph obtained by ignoring all the twists in $G$. By construction the natural isotopy for ribbon graphs is regular isotopy.

Remark. A ribbon graph is sometimes referred to as a framed tangle diagram.

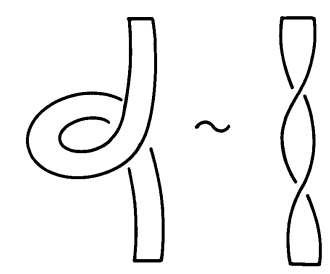

Figure 16.

Definition 3.2.2. An isotopy of ribbon graphs, denote by $[G]_{\mathrm{reg}}$, is an equivalence class of ribbon graphs generated by Reidemeister moves IIa, IIb and III on flat sections of the ribbon.

Definition 3.2.3. Let $G$ be an $l$-component ribbon graph with $n_{i}^{+}$clockwise twists and $n_{i}^{-}$counterclockwise twists on the $i$ th-component. Define a functor $\mathcal{V}_{\mathrm{RG}}$ acting on ribbon graphs by

$$
\mathcal{V}_{\mathrm{RG}}(G)=\left(\otimes_{i=1}^{l} \lambda^{\left(n_{i}^{-}-n_{i}^{+}\right) / 2}\right) \mathcal{V}\left(T_{G}^{*}\right) .
$$

Corollary 3.2. The functor $\mathcal{V}_{\mathrm{RG}}:\{G\} \rightarrow \mathcal{U}$ by $\mathcal{V}_{\mathrm{RG}}[G]_{\mathrm{reg}}=\mathcal{V}_{\mathrm{RG}}(G)$ is an invariant for ribbon graphs. 
Proof. $\mathcal{V}$ preserves writhes on $T_{G}$. In particular, the replacement of (2.4) by (3.10) equates a positive (negative) writhe, or a full clockwise (counterclockwise) twist, in a ribbon graph to a factor of $\lambda^{-1 / 2}\left(\lambda^{1 / 2}\right)$ in $\mathcal{V}$. Hence the extra factor of $\left(\otimes_{i} \lambda^{\left(n_{i}^{-}-n_{i}^{+}\right) / 2}\right)$ for $\mathcal{V}_{\mathrm{RG}}$ in (3.14).

Remark. Another terminology for a ribbon graph is a framed tangle. It is clear that if the central element $\lambda$ were equal to the identity element, then there would be no distinction between the $\mathcal{U}$ images of regular isotopic tangles, ambient isotopic tangles and ribbon graphs.

\subsection{Realization of $\mathcal{V}$ on representations of $\mathcal{U}$}

Let $V$ be an finite dimensional, irreducible $\mathcal{A}$ module and let $\pi_{i} \in \operatorname{End}(V)$. Then, from Schur's lemma, $\pi_{i}(\lambda)$ is equal to an eigenvalue $\lambda_{\pi_{i}}$ times the unit matrix. For an $l$-component tangle, a natural realization of $\mathcal{V}$ (or $\mathcal{V}_{A}$ or $\left.\mathcal{V}_{\mathrm{RG}}\right)$ valued on $\mathcal{A n l}$ is $\left(\pi_{1} \otimes \cdots \otimes \pi_{l}\right) \mathcal{V}$, in which $\lambda$ on the $i$ th string is represented by $\pi_{i}(\lambda)$, the $\mathcal{R}$-matrix at the crossing of the $i$ th and $j$ th strings is represented by $\mathcal{R}_{\pi_{i} \pi_{j}}=\left(\pi_{i} \otimes \pi_{j}\right) \mathcal{R}$, and so on, and $S p$ is replaced by the matrix trace in $\pi_{i}$. If $q$ takes value on $C$ (as opposed to being an indeterminate) then we restrict it to values such that $\lambda_{\pi_{i}} \neq 0$.

A case of special simplicity and interest is to let all $\pi_{i}=\pi$. In this case, write:

Definition 3.3.1. $\quad \lambda_{\pi}=\pi(\lambda), h_{\pi}=\pi(v), \mathcal{R}_{p} i=(\pi \otimes \pi) \mathcal{R}, \mathcal{R}_{p} i^{\prime}=(\pi \otimes \pi) \mathcal{T R}^{-1}$, and let $T r_{\pi}$ be the matrix trace in $\pi$.

Then, by replacing $\left\{\mathcal{R}, \mathcal{R}^{\prime}, v, v^{-1}, \lambda, S p\right\}$ by $\left\{\mathcal{R}_{p} i, \mathcal{R}_{p} i^{\prime}, h_{\pi}, h_{\pi}^{-1}, \lambda_{\pi}, T r_{\pi}\right\}$ in $\mathcal{V}$ $\left(\mathcal{V}_{A}, \mathcal{V}_{\mathrm{RG}}\right.$, reps. $)$, we get $\mathcal{V} \pi\left(\mathcal{V}_{A_{\pi}}, \mathcal{V}_{R G_{\pi}}\right.$, resp.).

\section{Universal tangle invariants and commutant of $\mathcal{U}$}

\subsection{The main proposition}

In this section we discuss commutants of $\mathcal{U}$. Since $\lambda$ is a central element of $\mathcal{U}$, we shall make no distinction between $\mathcal{V}, \mathcal{V}_{A}$ and $\mathcal{V}_{\mathrm{RG}}$; an invariant will be generically referred to as $\mathcal{V}[T]$. Let $\mathcal{U}$ be a quantum algebra $\mathcal{U}_{q}(g)$ of section 3.0 whose comultiplication and antipode on the generators $\left\{k_{i} \equiv e^{H_{i} / 2}, k_{i}^{-1} \equiv e^{-H_{i} / 2}, X_{i}^{+}, X_{i}^{-} ; i=1, \ldots\right\}$ are, for convenience, given again:

$$
\begin{aligned}
& \triangle X_{i}^{ \pm}=X_{i}^{ \pm} \otimes k_{i}+k_{i}^{-1} \otimes X_{i}^{ \pm} \\
& S\left(k_{i}^{ \pm 1}\right)=k_{i}^{\mp 1} \quad S\left(X_{i}^{ \pm}\right)=-k_{i} X_{i}^{ \pm} k_{i}^{-1} .
\end{aligned}
$$

Let $\mathcal{U}_{\triangle}$ be the subset of $\mathcal{U}$ generated by $\triangle$ acting on $\mathcal{A}, \mathcal{V}$ be the functor of section 3.1 valued on $\mathcal{U}, \sigma: T^{(n)} \rightarrow S_{n}$ be the natural mapping of tangle diagrams to the symmetry groups, $\triangle^{\prime\{n\}}: \mathcal{A} \rightarrow \mathcal{A}^{\otimes(n+1)}$ be the natural extension of $\triangle: \mathcal{A} \rightarrow \mathcal{A} \otimes \mathcal{A}$ (see definition 4.2.2). For $a_{1}, \ldots, a_{n} \in \mathcal{A}$, define $\mathcal{T}_{n} \in S_{n}$ by $\mathcal{T}_{n}\left(a_{1} \otimes a_{2} \otimes \cdots \otimes a_{n}\right)=a_{n} \otimes \cdots \otimes a_{2} \otimes a_{1}$, and $\Delta^{\prime\{n\}}=\mathcal{T}_{n+1} \circ \triangle^{\{n\}}$. Recall that if $T^{(n)}$ has $l$ closed strands, then $\mathcal{V}\left[T^{(n)}\right]=\alpha^{(n)} \otimes \alpha_{0}^{(l)}$, where a suppressed summation is understood, $\alpha^{(n)} \in \mathcal{A} n n, \alpha_{0}^{(l)} \in \mathcal{A} z l, \mathcal{A}_{0}=\mathcal{A} /\{(a b-b a) \mid a, b \in \mathcal{A}\}$.

Proposition 4. For an $(n, n)$-tangle diagram $T$ with $l$ closed strands,

$\left(\triangle^{\prime\{n-1\}}(a) \otimes 1^{\otimes l}\right) \mathcal{V}[T]=\mathcal{V}[T]\left(\sigma(T)\left(\triangle^{\prime\{n-1\}}(a)\right)\right) \otimes 1^{\otimes l} \quad \forall a \in \mathcal{A}$.

Suppose $\Delta^{\{n-1\}}(a)=\sum_{i} x_{1 i} \otimes x_{2 i} \otimes \cdots \otimes x_{n i} \equiv \otimes_{r=1}^{n} x_{r}, \alpha^{(n)}=\otimes_{r=1}^{n} t_{r}$ and $\sigma(T)$ sends $(1,2, \cdots, n)$ to $\left(k_{1}, k_{2}, \cdots, k_{n}\right)$. Then $\triangle^{\prime\{n-1\}}(a)=\otimes_{r=1}^{n} x_{r^{\prime}}$, where $r^{\prime}=n-r+1$, and (4.2) implies $\left(\otimes_{r=1}^{n} x_{r^{\prime}} t_{r}\right) \otimes \alpha_{0}^{(l)}=\left(\otimes_{r=1}^{n} t_{r} x_{k_{r}}\right) \otimes \alpha_{0}^{(l)}$. 


\subsection{A tangle Casimir operator}

We first give an example of what we shall call a tangle Casimir operator of $\mathcal{U}$. Let $T_{\text {trefoil }}$ be the $(1,1)$-tangle diagram shown in figure $17(a)$ whose closure is the trefoil link.

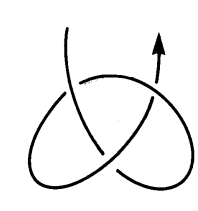

(a)

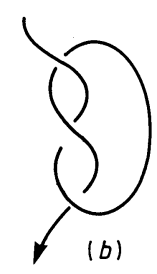

(b)

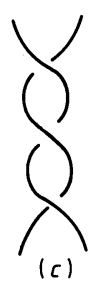

(c)

Figure 17.

Lemma 4.1.1. $\mathcal{V}\left[T_{\text {trefoil }}\right]$ valued in $\mathcal{U}_{q}(s l(2))$ is a central element.

Proof. The rank of $s l(2)$ is one, so the elements $H, X^{ \pm}, k$ no longer need subscripts. We simply write $X$ for $X^{ \pm}$. From (4.1),

$$
S^{2}\left(k^{ \pm 1}\right)=k^{ \pm 1} \quad S^{2}(X)=k^{2} X k^{-2} .
$$

Applying the rules given in section 2.4 on figure $17(b)$ we get

$$
\tau \equiv \mathcal{V}\left[T_{\text {trefoil }}\right]=A B^{\prime} A^{\prime \prime} v B A^{\prime} B^{\prime \prime} \equiv m((1 \otimes v) \beta)
$$

where $\beta=\mathcal{R}(\mathcal{T} \mathcal{R}) \mathcal{R}$ is the value of a two-strand braid shown in figure 17(b) whose partial closure is $T_{\text {trefoil }}$. The lemma is proven when it is shown that $\left[\tau, k^{ \pm 1}\right]=0$ and $[\tau, X]=0$. From (3.1c), $\beta$ commutes with $\Delta\left(k^{ \pm 1}\right)=k^{ \pm 1} \otimes k^{ \pm 1}$. For $\alpha \in \mathcal{A} A$ and $x, y, z \in \mathcal{A}$, $\beta \triangle(x)=\Delta^{\prime}(x) \beta$ and $m((x \otimes y z) \alpha)=m((x \otimes z) \alpha(y \otimes 1))$. Also $k, v$ commute, because $v k v^{-1}=S^{-2}(k)=k$. We first show $[\tau, k]=0$.

$$
\begin{array}{r}
\tau k=m((1 \otimes v) \beta(1 \otimes k))=m\left(\left(1 \otimes k k^{-1} v\right) \beta(1 \otimes k)\right)=m\left(\left(1 \otimes k^{-1} v\right) \beta \triangle(k)\right) \\
=m\left(\left(1 \otimes k^{-1} v\right) \triangle(k) \beta\right)=m\left(\left(k \otimes k^{-1} v k\right) \beta\right)=m((k \otimes v) \beta)=k \tau .
\end{array}
$$

We now show $[\tau, X]=0$.

$$
\tau X=m\left(\left(1 \otimes k k^{-1} v\right) \beta(1 \otimes X)\right)=m\left((1 \otimes k v) \beta\left(k^{-1} \otimes X\right)\right) .
$$

After adding and subtracting a term $m((1 \otimes X k v) \beta(1 \otimes k))=m((1 \otimes k v) \beta(X \otimes k))$ from the right-hand side, and using manipulations similar to those used in (4.3), one obtains

$$
\begin{aligned}
& \tau X=m\left((1 \otimes k v) \triangle^{\prime}(X) \beta\right)-m((1 \otimes X k v) \beta(1 \otimes k)) \\
& \quad=m\left(\left(X \otimes k v k^{-1}\right) \beta\right)+m((k \otimes k v X) \beta)-m((1 \otimes X k v) \beta(1 \otimes k)) .
\end{aligned}
$$

The first term on the right-hand side is just $X \tau$. The second term may be rewritten as

$m\left(\left(k \otimes k v X k^{-1} k\right) \beta\right)=m\left(\left(1 \otimes k v X k^{-1}\right) \triangle(k) \beta\right)=m\left(\left(1 \otimes k^{2} v X k^{-1}\right) \beta(1 \otimes k)\right)$

which, with the aid of (3.5) and (4.3), cancels the last term in (4.6b):

$$
k^{2} v X k^{-1}-X k v=k^{2} S^{-2}(X) v k^{-1}-X k v=0 .
$$

Therefore $[\tau, X]=0$. 
Remark. (4.7a), or $\left[k^{2} v, X\right]=0$, and $\left[k^{-2} v^{-1}, X\right]=0$ written in the form

$$
k^{-2} v^{-1} X k-X k^{-1} v^{-1}=0
$$

used in the context of counterclockwise and clockwise Seifert circles, respectively, are the canonical relations needed to prove proposition 4. The following generalizes lemma 4.1.1 to any quantum algebra of section 3.0.

Lemma 4.1.2. $\mathcal{V}\left[T_{\text {trefoil }}\right]$ valued on any $\mathcal{U}$ is a central element.

Proof. For any $\mathcal{U},(4.1)$ assures that (4.7a) and (4.7b), with $k^{ \pm 1}, X$ substituted by $k_{i}^{ \pm 1}, X \pm_{i}$, are true.

\subsection{A graphical approach}

In the rest of section 4 , unless otherwise stated, $k$ denotes $k_{i}$ and $X$ denotes either $X_{i}^{+}$or $X_{i}^{-}$.

Definition 4.2.0. Extend the graphical representation by arrows for $\mathcal{V}[T]$ valued on $\mathcal{U}$, given in section 2.4, to a graphical representation for the entire $\mathcal{U}$ as follows. As before, the graphical representation of $\mathcal{V}\left(T^{*}\right) \in \mathcal{U}$ is given by the graphical representation of the specified tangle diagram $T^{*}$, that is, the components $1, v, v^{-1}, \mathcal{R}, \mathcal{R}^{\prime}$ in $\mathcal{V}\left(T^{*}\right)$ are respectively represented by the arrows $\downarrow, \downarrow, \downarrow, \downarrow, \downarrow$. Furthermore, let the arrows $\hat{\phi}, \downarrow$ and $\downarrow$ respectively represent $k, k^{-1}$ and $X$. Call an element in $\mathcal{U}$ thus represented a tangle graph. The relative direction of an arrow in a tangle graph carries no significance.

Definition 4.2.1. Define a graphical representation of $\mathcal{U}$ using the same arrows given above, except to represent $\mathcal{V}\left(T^{*}\right)$ by the splice of $T^{*}$. Specifically, use the symbols $2+\left(, L^{-}-\right.$, respectively, to represent $\mathcal{R}, \mathcal{R}^{\prime}$, respectively, and use the symbol ${ }^{*}\left(\right.$ to represent $\mathcal{R}^{*}$ that denotes either $\mathcal{R}$ or $\mathcal{R}^{\prime}$. Call an element in $\mathcal{U}$ thus represented a splice graph.

Remark. The set of tangle diagrams (splices, resp.) is a subset of tangle graphs (splice graphs, resp.).

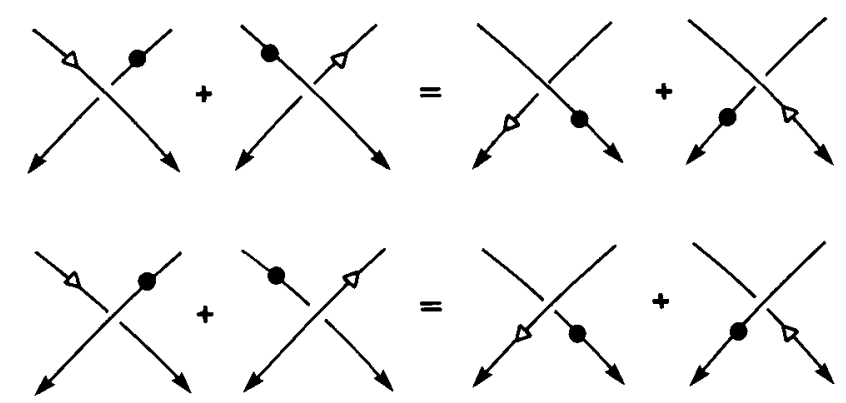

Figure 18.

Example. The coboundary relations $\Delta^{\prime}(X) \mathcal{R}^{*}=\mathcal{R}^{*} \Delta(X)$ are expressed in terms of tangle graphs in figure 18 and splice graphs in figure 19. Observe that the two sets of splice graphs in figure 19 are united as one in figure 20 when the symbol ${ }^{*}$ is used to represent $\mathcal{R}^{*}$. 

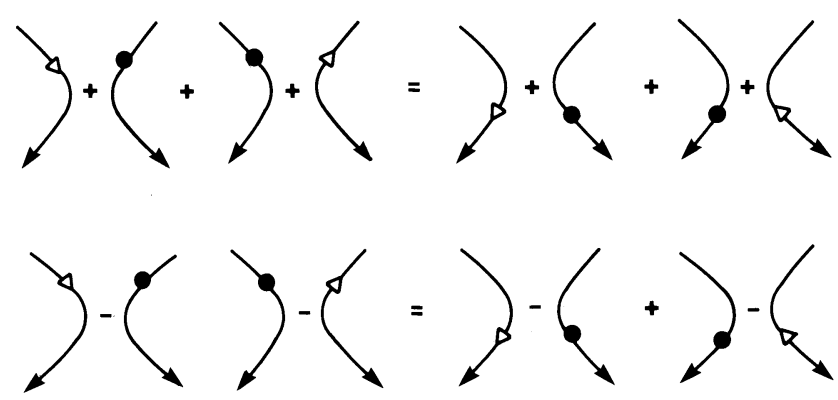

Figure 19.

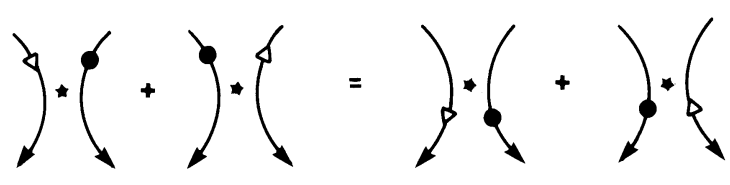

Figure 20.

Remark. Note that, in figure 20, the relations allow the arrows representing $k, k^{-1}$ and $X$ to slide in union past the crossing (the star in figure 20) along the lines on which they live. This comes about because on a spliced crossing, the tensor spaces represented by the two lines are transposed at the crossing. Generally, if a tensor $\alpha \in \mathcal{A} \otimes \mathcal{A}$ can slide past a crossing on a splice graph, then $\alpha \mathcal{R}^{*}=\mathcal{T}\left(\left(\mathcal{T}^{*}\right) \alpha\right)=\mathcal{R}^{*} \mathcal{T} \alpha$. A generalized version of this observation is given as lemma 4.2.1.

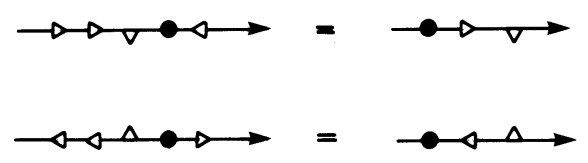

Figure 21.

Example. Figure 21 $(a)$ and $(b)$ are graphical expressions of the relations $(4.7 a, b)$.

Example. Figure 22 shows a proof of $\tau X=\tau X$ using splice graphs. Figure 19 is used to obtain the second line of the figure, while figure 21 is used to obtain the last line.

Definition 4.2.2. Let $\Delta^{\{1\}}=\Delta$. Define, for $n \geqslant 2$,

$$
\begin{aligned}
& \Delta^{\{n\}} \equiv(\mathrm{id} \otimes \mathrm{id} \cdots \otimes \triangle) \triangle^{\{n-1\}}=\left(\mathrm{id}^{\otimes(n-1)} \otimes \triangle\right) \triangle^{\{n-1\}} \\
& \mathcal{A}^{\{n\}} \equiv \triangle^{\{n-1\}} \mathcal{A} \in \mathcal{U} \triangle \subset \mathcal{U} .
\end{aligned}
$$

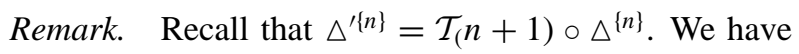

$\Delta^{\{n\}} k^{ \pm 1}=\Delta^{\{n\}} k^{ \pm 1}=k^{ \pm 1 \otimes(n+1)}$

$\Delta^{\{n\}} X=\sum_{\nu=0}^{n} k^{-1^{\otimes v}} \otimes X \otimes k^{\otimes(n-v)} \quad \Delta^{\{n\}} X=\sum_{\nu=0}^{n} k^{\otimes v} \otimes X \otimes k^{-1^{\otimes(n-v)}}$. 

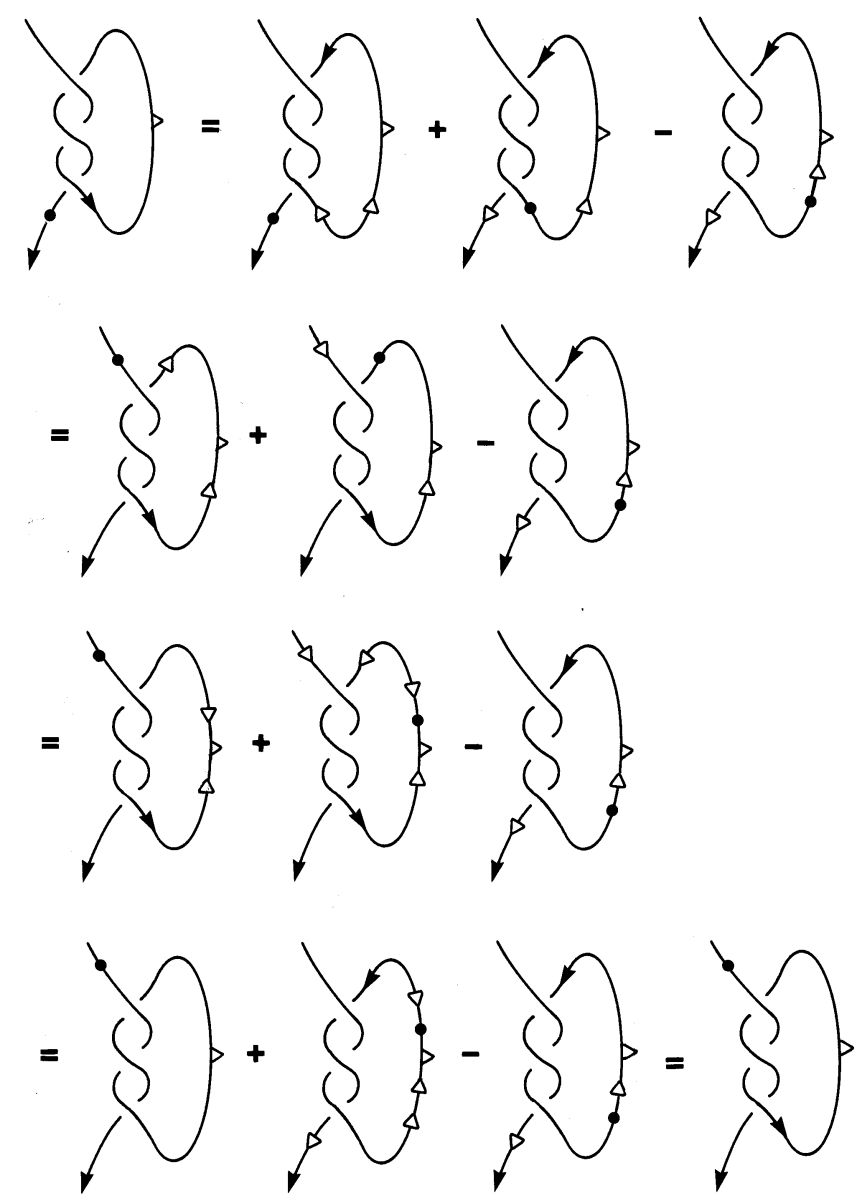

Figure 22.

Lemma 4.2.1. For any braid $\mathcal{B} \in B_{n}, a \in \mathcal{A}$,

$$
\left(\Delta^{\prime\{n-1\}}(a)\right) \mathcal{V}(\mathcal{B})=\mathcal{V}(\mathcal{B})\left(\sigma(\mathcal{B}) \circ \Delta^{\prime\{n-1\}}(a)\right) .
$$

Proof. The lemma is proved if (4.12) is proven for $a=k^{ \pm 1}$ and $X$. The relation is trivially true for $a=k^{ \pm 1}$, because $\Delta\left(k^{ \pm 1}\right)$ commute with $\mathcal{R}^{*}$. For $a=X$, first consider the case when $\mathcal{B}=b_{i}^{ \pm}$in which only the $i$ th and $(i+1)$ st strands are braided once. Then $\mathcal{V}\left(b_{i}^{ \pm}\right)=\mathcal{R}_{i, i+1}^{*}$. Noting that $\sigma(b) \circ \Delta^{\prime}=\Delta$ when $b \in B_{2}$ is the standard braid generator, we write

$$
\sigma\left(b_{i}^{ \pm}\right) \circ \Delta^{\prime\{n-1\}}(X)=\sum_{v \neq i-1, i} k^{\otimes v} \otimes X \otimes k^{-1^{\otimes(n-v)}}+k^{\otimes(i-1)} \otimes \Delta(X) \otimes k^{-1^{\otimes(n-i)}}
$$

therefore $\Delta^{\prime\{n-1\}}(X) \mathcal{V}\left(b_{i}^{ \pm}\right)=\mathcal{V}\left(b_{i}^{ \pm}\right)\left(\sigma\left(b_{i}^{ \pm}\right) \circ \Delta^{\prime\{n-1\}}(X)\right)$. Repeated application of this relation gives (4.12). The composition $\sigma(\mathcal{B}) \circ \Delta^{\prime n-1\}}$ ensures that $\mathcal{V}(\mathcal{B})$ acts on the correct tensor spaces on the right-hand side of (4.12).

Example. How $\triangle^{\prime\{n-1\}}(X)$ gets past the first crossing in $\mathcal{V}(\mathcal{B})$ is shown on splice graphs in figure 23. Getting past subsequent crossings is achieved by repeating the same process. 

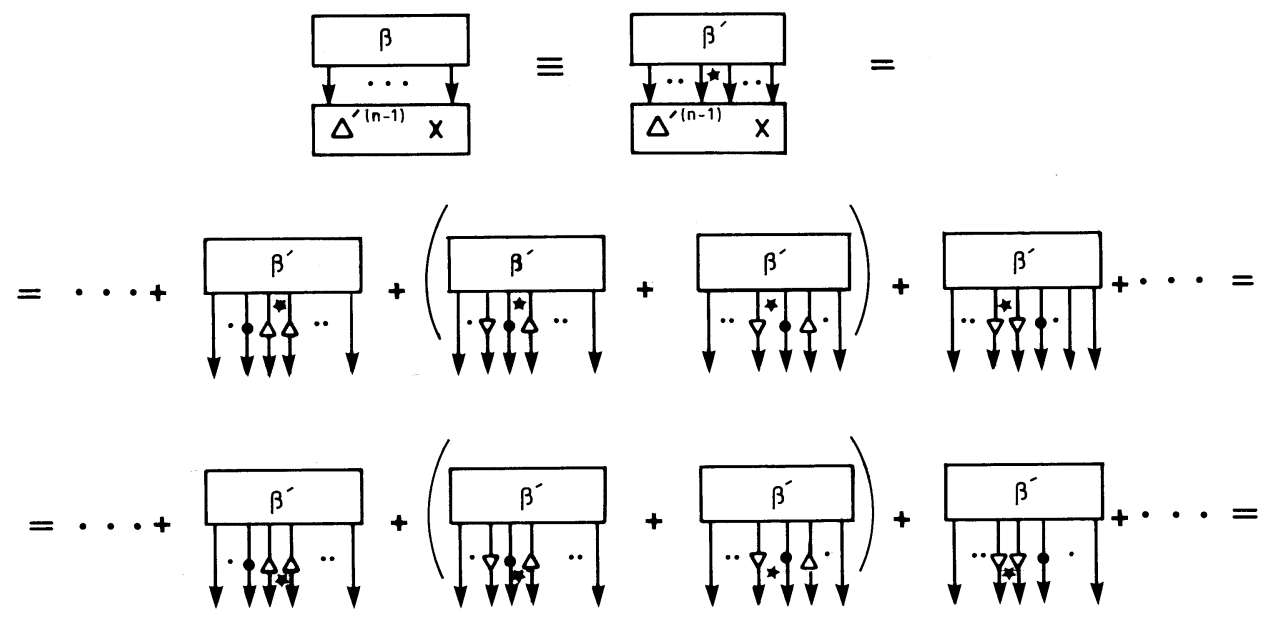

$$
=\frac{\beta^{\prime}}{\frac{\beta^{\prime} \cdot 1}{\Delta \Delta^{(n-1)} x}}
$$

Figure 23.

\subsection{Proof of proposition 4}

$T$ is an $(n, n)$-tangle diagram with $l$ closed strands. We use the notation of sections 2.5.2 and 2.5.3, where $\mathcal{B}$ is a braid of $N=n+\mu_{\mathrm{L}}+\mu_{\mathrm{R}}$ strands whose partial closure is a braidtangle diagram $T_{\mathcal{B}}$ that is isotopic to $T, \beta \equiv \mathcal{V}(\mathcal{B}) \in \mathcal{A} n N$ and $\mathcal{H} \equiv v^{-1 \otimes \mu_{\mathrm{L}}} \otimes 1^{\otimes n} \otimes v^{\otimes \mu_{\mathrm{R}}}$. We first derive two extensions of (2.17).

Lemma 4.3.1 For $\alpha^{\prime}=1^{\otimes \mu_{\mathrm{L}}} \otimes \alpha \otimes 1^{\otimes \mu_{\mathrm{R}}} \in \mathcal{A} n N, \forall \alpha \in \mathcal{A} n n$ :

$$
\begin{aligned}
& \mathcal{V}\left[T_{\mathcal{B}}\right]\left(\alpha \otimes 1^{\otimes l}\right)=C l\left\{T_{\mathcal{B}}\right\}\left(\mathcal{H} \beta \sigma(\mathcal{B})\left(\alpha^{\prime}\right)\right)=\operatorname{Cl}\left\{T_{\mathcal{B}}\right\}\left(\beta \sigma(\mathcal{B})\left(\alpha^{\prime} \mathcal{H}\right)\right) \\
& \left(\alpha \otimes 1^{\otimes l}\right) \mathcal{V}\left[T_{\mathcal{B}}\right]=C l\left\{T_{\mathcal{B}}\right\}\left(\alpha^{\prime} \mathcal{H} \beta\right)=C l\left\{T_{\mathcal{B}}\right\}\left(\alpha^{\prime} \beta \sigma(\mathcal{B})(\mathcal{H})\right) .
\end{aligned}
$$

Lemma 4.3.2. For $\beta^{\prime} \in \mathcal{A}^{\otimes N}$ and $\gamma=\gamma_{\mathrm{L}} \otimes 1^{\otimes n} \otimes \gamma_{\mathrm{R}} \in \mathcal{A}^{\otimes N}, \forall \gamma_{\mathrm{L}} \in \mathcal{A} n \mu_{\mathrm{L}}, \gamma_{\mathrm{R}} \in \mathcal{A} n \mu_{\mathrm{R}}$ :

$$
C l\left\{T_{\mathcal{B}}\right\}\left(\gamma \beta \sigma(\mathcal{B})\left(\beta^{\prime}\right)\right)=C l\left\{T_{\mathcal{B}}\right\}\left(\beta \sigma(\mathcal{B})\left(\beta^{\prime} \gamma\right)\right) .
$$

Proof. The lemmas follow directly from the property of $C l\left\{T_{\mathcal{B}}\right\}$. We omit details.

Remark. The right-hand sides of (4.13) and (4.14) are not supposed to represent any topological object.

Proof of proposition 4. The proposition is proven when it is shown that (4.2) is satisfied for $a=k^{ \pm 1}$ and $X$. The proof for $a=k^{ \pm 1}$ follows simply from lemma 4.2.1 and the fact that $k^{ \pm 1}$ commute with $v$ and $v^{-1}$; we omit details. 
We now prove the case for $a=X$. Let $X^{\{s\}} \equiv \triangle^{\{\{s-1\}}(X)$ and define the following elements $\in \mathcal{A}^{\otimes N}$, where $\left(k^{ \pm 1}\right)^{\otimes t} \equiv 1$ if $t=0$ :

$X^{\prime\{n\}} \equiv 1^{\otimes \mu_{\mathrm{L}}} \otimes X^{\{n\}} \otimes 1^{\otimes \mu_{\mathrm{R}}} \quad \mathcal{Q} \equiv\left(k^{-1}\right)^{\otimes \mu_{\mathrm{L}}} \otimes 1^{\otimes n} \otimes k^{\otimes \mu_{\mathrm{R}}}$

$\mathcal{W}_{(t)} \equiv k^{\otimes(t-1)} \otimes X \otimes\left(k^{-1}\right)^{\otimes(N-t)} \quad \mathcal{W}_{\mathrm{L}} \equiv \sum_{t=1}^{\mu_{\mathrm{L}}} \mathcal{W}_{(t)} \quad \mathcal{W}_{\mathrm{R}} \equiv \sum_{t=N-\mu_{\mathrm{R}}+1}^{N} \mathcal{W}_{(t)}$

$\mathcal{W}_{\mathrm{L}}^{\prime} \equiv \mathcal{W}_{\mathrm{L}} \cdot k^{\otimes N} \quad \mathcal{W}_{\mathrm{R}}^{\prime} \equiv \mathcal{W}_{\mathrm{R}} \cdot\left(k^{-1}\right)^{\otimes N}$

Then,

$$
X^{\{N\}} N=X^{\prime\{n\}} \mathcal{Q}^{-1}+\mathcal{W}_{\mathrm{L}}+\mathcal{W}_{\mathrm{R}}
$$

and from lemma 4.2.1,

$$
\begin{aligned}
\beta \sigma(\mathcal{B})\left(X^{\{N\}} \mathcal{Q}\right) & =X^{\{N\}} \beta \sigma(\mathcal{B})(\mathcal{Q}) \\
& =\mathcal{Q}^{-1} X^{\prime\{n\}} \beta \sigma(\mathcal{B})(\mathcal{Q})+\mathcal{W}_{\mathrm{L}}^{\prime} \beta \sigma(\mathcal{B})\left(\left(k^{-1}\right)^{\otimes N} \mathcal{Q}\right)+\mathcal{W}_{\mathrm{R}}^{\prime} \beta \sigma(\mathcal{B})\left(k^{\otimes N} \mathcal{Q}\right) .
\end{aligned}
$$

Observe that $\mathcal{Q}, \mathcal{H}$ and $X^{\prime\{n\}}$ commute among themselves, and that $\mathcal{H}, \mathcal{Q}, \mathcal{W}_{\mathrm{L}}^{\prime}$ and $\mathcal{W}_{\mathrm{R}}^{\prime}$ all have the form of $\gamma$ in (4.14). Now use (4.13)-(4.16) to write

$$
\begin{aligned}
&\left(X^{\{n\}} \otimes 1^{\otimes l}\right) \mathcal{V}\left[T_{\mathcal{B}}\right]-\mathcal{V}\left[T_{\mathcal{B}}\right]\left(\sigma\left(T_{\mathcal{B}}\right)\left(X^{\{n\}}\right)\right) \otimes 1^{\otimes l} \\
&= C l\left\{T_{\mathcal{B}}\right\}\left(X^{\prime\{n\}} \beta \sigma(\mathcal{B})(\mathcal{H})\right)-C l\left\{T_{\mathcal{B}}\right\}\left(\mathcal{H} \beta \sigma(\mathcal{B})\left(X^{\prime\{n\}}\right)\right) \\
&= C l\left\{T_{\mathcal{B}}\right\}\left(\beta \sigma(\mathcal{B})\left(\mathcal{W}_{\mathrm{L}} \mathcal{Q H}-\left(k^{-1}\right)^{\otimes N} \mathcal{Q H} \mathcal{W}_{\mathrm{L}}^{\prime}+\mathcal{W}_{\mathrm{R}} \mathcal{Q H}-k^{\otimes N} \mathcal{Q H} \mathcal{W}_{\mathrm{R}}^{\prime}\right)\right) \\
&= C l\left\{T_{\mathcal{B}}\right\}\left(\beta \sigma ( \mathcal { B } ) \left(\sum_{t=1}^{\mu_{\mathrm{L}}}\left(v^{-1}\right)^{\otimes(t-1)} \otimes\left(X k^{-1} v^{-1}-k^{-2} v^{-1} X k\right) \otimes\left(k^{-2} v^{-1}\right)^{\otimes\left(\mu_{\mathrm{L}}-t\right)}\right.\right. \\
& \otimes\left(k^{-1}\right)^{\otimes n} \otimes v^{\otimes \mu_{\mathrm{R}}}+\sum_{t=1}^{\mu_{\mathrm{R}}}\left(v^{-1}\right)^{\otimes \mu_{\mathrm{L}}} \otimes k^{\otimes n} \otimes\left(k^{2} v\right)^{\otimes(t-1)} \otimes\left(X k v-k^{2} v X k^{-1}\right) \\
&\left.\left.\otimes v^{\otimes\left(\mu_{\mathrm{R}}-t\right)}\right)\right)=0 .
\end{aligned}
$$

The last equality follows from (4.7). Since $\mathcal{V}\left[T_{\mathcal{B}}\right]=\mathcal{V}[T]$ and $\sigma\left(T_{\mathcal{B}}\right)=\sigma(T)$, the proposition is proven.

Example. The proof is reproduced in figure 24 on splice graphs for the case $n=\mu_{\mathrm{L}}=$ $\mu_{\mathrm{R}}=1$.

\subsection{Commutants of $\mathcal{U}_{\triangle}$}

Owing to the appearance of permutations $\sigma(T)$ and $\mathcal{T}_{n}$ in proposition 4, the elements $\mathcal{V}[T]$ do not quite commute with $\mathcal{U}_{\triangle}$. Noting that $\mathcal{V}$ sends each closed strand in $T$ to $\mathcal{A}_{0}$, which commutes with $\mathcal{U}_{\triangle}$, in the following, without loss of generality, we consider only tangle diagrams with no closed strands. 


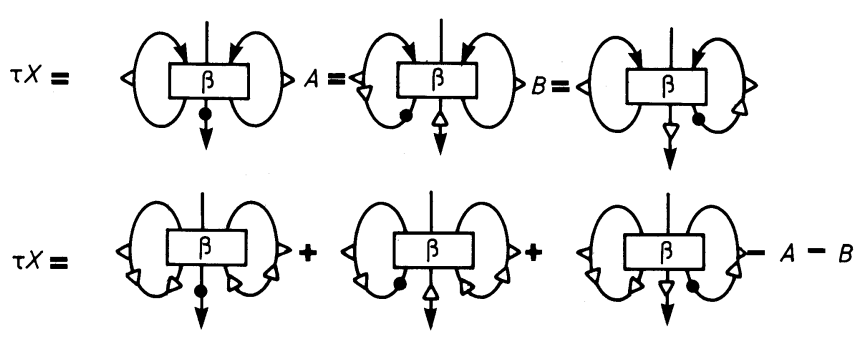

$=\left\{\frac{1}{D^{\prime \prime 2} x}>-A-B=\left\{\frac{\Delta^{\prime 21} x}{3}>-A-B\right.\right.$

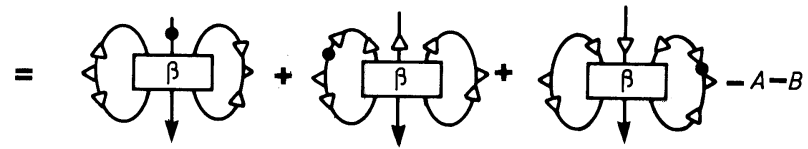

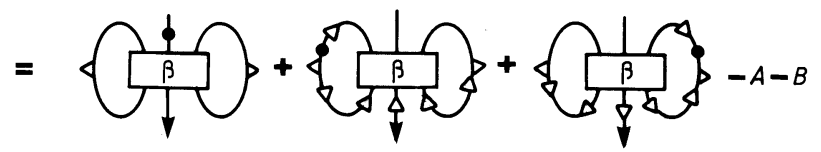

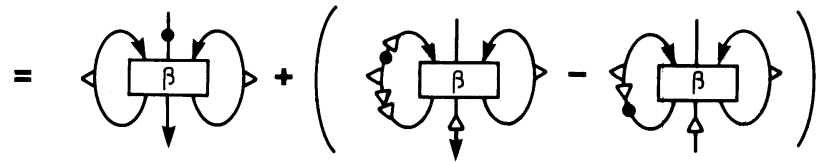

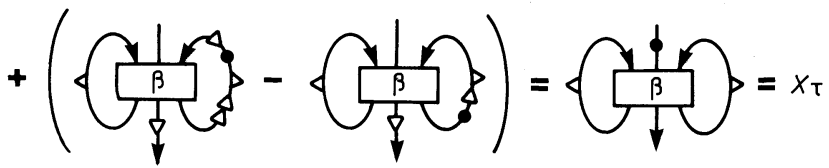

Figure 24.

Corollary 4.1. For $T \in T^{(n)}$ such that $\sigma(T)$ is the identity element in $S_{n}, \mathcal{T}_{n} \mathcal{V}[T]$ lies in the commutant of $\mathcal{U}_{\triangle}$ :

$$
\left[\mathcal{T}_{n} \mathcal{V}[T], \Delta^{\{n-1\}}(\mathcal{A})\right]=0
$$

Proof. This follows simply from proposition 4. Note that here $\mathcal{T}_{n}$ acts on $\mathcal{V}$ instead of acting on $\Delta^{\{n-1\}}$.

Example. For the special case $n=2$, with $T$ being a $(2,2)$-tangle diagram with two positive crossings, $\mathcal{V}[T]=\mathcal{T}(\mathcal{R}) \mathcal{R}$ commutes with $\Delta(\mathcal{A})$.

Remark. For $n=1$, the corollary means that the set of all $\mathcal{V}\left[T^{(1)}\right]$ lives in the centre of $\mathcal{A}$. For $n>1$, the set of all $\mathcal{T}_{n} \mathcal{V}\left[T^{(n)}\right]$ lives in $\mathcal{A} n n$ but not necessarily in $\Delta^{\{n-1\}}(\mathcal{A}) \subset \mathcal{A} n n$, hence it does not form a centre of $\Delta^{\{n-1\}}(\mathcal{A})$. In particular, two different $\mathcal{V}\left[T^{(n)}\right]$ 's in general do not commute. 
4.5. $\mathcal{V}$ maps $(1,1)$-tangles to the centre of $\mathcal{U}$

Corollary 4.2. $\mathcal{V}$ maps one-component $(1,1)$-tangles to the centre of $\mathcal{A}$.

Proof. This follows trivially from proposition 4.

Remark. In particular, the images of two one-component $(1,1)$-tangles commute. This can also be seen in the following way. Let $T$ and $T^{\prime}$ be two such tangles and let $\alpha=\mathcal{V}[T]$ and $\alpha^{\prime}=\mathcal{V}\left[T^{\prime}\right]$. Then $T^{\prime} T$ and $T T^{\prime}$ are two $(1,1)$-tangles that are isotopic: $T^{\prime} T \sim T T^{\prime}$. By definition $\mathcal{V}\left(\left(T^{\prime} T\right)^{*}\right)=\mathcal{V}\left(T^{\prime *}\right) \mathcal{V}\left(T^{*}\right)=\alpha^{\prime} \alpha$, and $\mathcal{V}\left(\left(T T^{\prime}\right)^{*}\right)=\alpha \alpha^{\prime}$. Therefore $\left[\alpha^{\prime}, \alpha\right]=0$.

One can naturally enlarge the centre to include the images of $(1,1)$-tangles with closed strands. Consider the set $\{T\}_{l}$ of all $(1,1)$-tangles with $l-1$ closed strands. Then $\mathcal{V}:\{T\}_{l} \rightarrow \mathcal{A} \otimes \mathcal{A} z(l-1) \equiv \mathcal{A}_{l} \subset \mathcal{A} n l$. By construction $\mathcal{A}_{0}$ commutes with $\mathcal{A}$. Hence, we state without further proof:

Corollary 4.3. $\mathcal{V}$ maps the set of all $(1,1)$-tangles to the centre of $\mathcal{U}$.

Remark. Valued on $\mathbb{C} \llbracket \eta \rrbracket$ with an $\eta$-adic topology, these central elements in principle can be expressed as polynomials of the quadratic Casimir operators of $\mathcal{U}$.

\subsection{Commutants of $\mathcal{U}_{\triangle}$ in a representation}

Definition 4.6. For a representation $\pi$ of $\mathcal{A}$ on a vector space $V$, define $\mathcal{A}_{\pi}^{\{n\}}=$ $\left(\pi^{\otimes n}\right)\left(\Delta^{\{n\}} \mathcal{A}\right)$. For an $(n, n)$-tangle $T, \sigma(T) \in S_{n}$ has a natural action on $V^{\otimes n}$. Define $\mathcal{V}_{\pi}(T)=\pi^{\otimes n}(\mathcal{V}[T]), \check{\mathcal{V}}_{\pi}(T)=\left(\left(\pi^{\otimes n}\right)\left(\mathcal{T}_{n} \mathcal{V}[T]\right)\right) \sigma(T)$.

Corollary 4.4. For every $T \in T^{(n)}$,

$$
\left[\check{\mathcal{V}}_{\pi}(T), \mathcal{A}_{\pi}^{\{n\}}\right]=0 .
$$

Proof. This is a direct consequence of proposition 4.

Remark. Thus, in a representation, $\check{\mathcal{V}}_{\pi}$ maps tangles to (representations of) commutants of $\mathcal{U}_{\triangle}$. Suppressing a summation, we write $\mathcal{V}[T]=\otimes_{i=1}^{n} u_{i} ; u_{i} \in \mathcal{A}$. Suppose the action of $\sigma(T)$ is such that $\sigma(T)\left(\otimes_{i=1}^{n} V_{i}\right)=\otimes_{i=1}^{n} V_{\sigma_{i}}$, where the subscripts label the order in the tensor product. Then the matrix elements of $\mathcal{V}_{\pi}(T)$ and $\breve{\mathcal{V}}_{\pi}(T)$ are given by

$$
\mathcal{V}_{\pi}(T)_{b_{1} b_{2} \ldots b_{n}}^{a_{1} a_{2} \ldots a_{n}}=\prod_{i=1}^{n}\left(\pi\left(u_{i}\right)\right)_{b_{i}}^{a_{i}} \quad \check{\mathcal{V}}_{\pi}(T)_{b_{1} b_{2} \ldots b_{n}}^{a_{1} a_{2} \ldots a_{n}}=\prod_{i=1}^{n}\left(\pi\left(u_{n-i+1}\right)\right)_{b_{\sigma_{i}}}^{a_{i}} .
$$

Example. Corresponding to the $\mathcal{R}$-matrix we have the familiar expression $\check{\mathcal{R}}_{r s}^{a b}=$ $\pi(A)_{r}^{b} \pi(B)_{s}^{a}$.

Example. Given a (3,3)-tangle $T$ with $\mathcal{V}[T]=U \otimes V \otimes W$ and braiding action $\sigma(T)(U \otimes V \otimes W)=W \otimes U \otimes V$, we have $\breve{\mathcal{V}}_{\pi}(T)_{r s t}^{a b c}=\pi(W)_{t}^{a} \pi(V)_{r}^{b} \pi(U)_{s}^{c}$. Suppose $\triangle^{\{2\}}(a)=X \otimes Y \otimes Z$. Then proposition 4 implies $Z U \otimes Y V \otimes X W=U Y \otimes V X \otimes W Z$, or, equivalently, $X W \otimes Y V \otimes Z U=W Z \otimes V X \otimes U Y$. Taking matrix elements and using the shorthand where $U$ stands for $\pi(U)$, we have

$$
\begin{aligned}
\sum_{e f g}(X \otimes Y \otimes Z)_{e f g}^{a b c}(W \otimes V \otimes U)_{r s t}^{e f g} & =\sum_{e f g}(W \otimes V \otimes U)_{e f g}^{a b c}(Z \otimes X \otimes Y)_{r s t}^{e f g} \\
& =\sum_{e f g}(W \otimes V \otimes U)_{e f g}^{a b c}(X \otimes Y \otimes Z)_{s t r}^{f g e}
\end{aligned}
$$


or, in terms of $\check{\mathcal{V}}_{\pi}$,

$$
\sum_{e f g}\left(\left(\pi^{\otimes n}\right)\left(\triangle^{\{n-1\}}(a)\right)\right)_{e f g}^{a b c}\left(\check{\mathcal{V}}_{\pi}(T)\right)_{s t r}^{e f g}=\sum_{e f g}\left(\check{\mathcal{V}}_{\pi}(T)\right)_{f g e}^{a b c}\left(\left(\pi^{\otimes n}\right)\left(\Delta^{\{n-1\}}(a)\right)\right)_{s t r}^{f g e}
$$

\section{Tangle Casimir operators and link polynomials}

Corollary 5. The eigenvalue of a tangle Casimir operator of $\mathcal{U}$ in an irreducible representation is an invariant, or link polynomial, of the closure of the tangle.

Let $V$ be an finite dimensional, irreducible $\mathcal{A}$ module, $\pi \in \operatorname{End}(V)$ and $\mathcal{V}_{\pi}$ (or $\mathcal{V}_{A \pi}$ ) be the invariant given in definition 3.3.1. Proposition 4 and corollaries 4.1, 4.2 and 4.3 apply to $\mathcal{V}_{\pi}$. Let $T$ be a $(1,1)$-tangle. By Schur's lemma, $\mathcal{V}_{\pi}[T]$ is equal to the eigenvalue $Q_{\pi}[T]$ times the unit matrix on $\pi$ :

$$
\mathcal{V}_{\pi}[T]=Q_{\pi}[T] \mathbf{1}_{\pi}
$$

That is, $Q_{\pi}[T]$ is the eigenvalue of tangle Casimir operator $\mathcal{V}[T]$ in the representation $\pi$.

Remark. Relation (5.1) was first conjectured in Lee et al (1988), Couture et al (1990) and Lee (1990).

We now prove corollary 5 by considering separately the cases $\operatorname{Tr}_{\pi}\left(h_{\pi}\right) \neq 0$ and otherwise.

Corollary 5.1. If $\operatorname{Tr}_{\pi}\left(h_{\pi}\right) \neq 0$, then $Q_{\pi}[T]$ is a link invariant for $[\hat{T}]$.

Proof. $\quad h_{\pi}$ and $h_{\pi}^{-1}$ are so defined ((3.5) and definitions 3.0.3 and 3.3.1) such that $\operatorname{Tr}_{\pi}\left(h_{\pi}\right)=$ $\operatorname{Tr}_{\pi}\left(h_{\pi}^{-1}\right)$. By definition $\mathcal{V}[\hat{T}]=\operatorname{Tr}_{\pi}\left(\mathcal{V}_{\pi}[T] h_{\pi}\right)=\operatorname{Tr}_{\pi}\left(\mathcal{V}_{\pi}[T] h_{\pi}^{-1}\right)=Q_{\pi}[T] \operatorname{Tr}_{\pi}\left(h_{\pi}\right)$ is an invariant for $[\hat{T}]$.

Remark. The usual link polynomial defined with a Markov trace is equivalent to $\mathcal{V}[\hat{T}]$. The above shows that, when $\operatorname{Tr}_{\pi}\left(h_{\pi}\right)=0$, the Markov trace maps all links trivially to zero.

Corollary 5.2. For non-trivial $\mathcal{V}_{\pi}, Q_{\pi}[T]$ is a link invariant for $[\hat{T}]$.

Remark. Non-triviality of $\mathcal{V}_{\pi}$ will be defined below. If $\operatorname{Tr}_{\pi}\left(h_{\pi}\right)=0$, then $\mathcal{V}_{\pi}[L]=0$ for all $[L]$. In this case corollary 5.1 is not useful since it is no longer possible to prove that $Q_{\pi}[T]$ is a link invariant by invoking (5.1).

Denote the set of all indecomposable representations of $\mathcal{U}$ by $\{\Lambda\}$ and let $\pi \in\{\Lambda\}$ be an irreducible representation. Let $T$ be a $(2,2)$-tangle for which $\sigma(T)=1 \in S_{2}$ and write $\mathcal{M}=\mathcal{V}_{\pi}(T)$. $\mathcal{M}$ has matrix elements $\mathcal{M}_{u t}^{r s}=\langle r, s|\mathcal{M}| t, u\rangle$ where $|t\rangle$ is a basis vector of $\pi,\langle t|$ is its dual and $|t, u\rangle=|t\rangle \otimes|u\rangle$. From corollary 4.1, $\mathcal{M}$ commutes with $\pi \otimes \pi(\Delta \mathcal{A})$. The tensor product representation $\pi \otimes \pi$ can be decomposed into

$$
\pi \otimes \pi=\Lambda_{1} \oplus \Lambda_{2} \oplus \cdots=\oplus_{a} \Lambda_{a} \quad \Lambda_{a} \in\{\Lambda\}
$$


and such a decomposition is unique up to a re-ordering of $\Lambda_{a}$. In each $\Lambda_{a}, \mathcal{M}$ acts as $\tau_{a}(T) \mathbf{1}_{a}$, where $\tau_{a}(T)$ is its eigenvalue. Therefore,

$$
\mathcal{M}=\sum_{a, i}\left|\Lambda_{a}^{i}\right\rangle \tau_{a}(T)\left\langle\Lambda_{a}^{i}\right|=\sum_{a} \tau_{a}(T) \mathbf{1}_{a}
$$

where $\left\langle\Lambda_{a}^{i}\right|$ form a basis of $\Lambda_{a}$ and $\left|\Lambda_{a}^{i}\right\rangle$ form a dual basis. Then,

$$
\mathcal{M}_{u t}^{r s}=\sum_{a, i}\left\langle r, s \mid \Lambda_{a}^{i}\right\rangle \tau_{a}(T)\left\langle\Lambda_{a}^{i} \mid t, u\right\rangle
$$

where $\left\langle\Lambda_{a}^{i} \mid t u\right\rangle$ is a Clebsch-Gordan coefficient. Let $T_{\mathrm{L}}\left(T_{\mathrm{R}}\right)$ be the $(1,1)$-tangle obtained by partially closing $T$ from the left (right). Then

$$
Q_{\pi}\left[T_{\mathrm{L}}\right]=\sum_{a} \xi_{\mathrm{L} a} \tau_{a}(T) \quad Q_{\pi}\left[T_{\mathrm{R}}\right]=\sum_{a} \xi_{\mathrm{R} a} \tau_{a}(T)
$$

where the coefficient

$$
\xi_{\mathrm{L} a}=\sum_{i, r, u}\left\langle r, s \mid \Lambda_{a}^{i}\right\rangle\left\langle r\left|h_{\pi}^{-1}\right| t\right\rangle\left\langle\Lambda_{a}^{i} \mid t, s\right\rangle
$$

depends on the quantum algebra and its representations but not on $T$. Furthermore, because of (5.1), it is independent of the unsummed index $s$. Similarly for $\xi_{\mathrm{R} a}$, for which $h_{\pi}^{-1}$ in (5.6) is replaced by $h_{\pi}$.

Let $n(\Lambda)$ be the number of indecomposable representations appearing in (5.3). Consider $T$ 's such that $T_{\mathrm{L}} \sim T_{\mathrm{R}}$. There is an infinite number of such $T$ 's whose $T_{\mathrm{L}}\left(\sim T_{\mathrm{R}}\right)$ are mutually non-isotopic. Suppose $T$ and $T^{\prime}$ are non-isotopic. We say $T$ is degenerate with $T^{\prime}$ with respect to $\pi$ if $\tau_{a}(T)=\tau_{a}\left(T^{\prime}\right)$ at least for one $\Lambda_{a}$. We define $\mathcal{V}_{\pi}$ as being non-trivial if the number of non-degenerate non-isotopic $T$ 's is greater than $n(\Lambda)$.

Lemma 5.1. For non-trivial $\mathcal{V}_{\pi}, Q_{\pi}\left[T_{\mathrm{L}}\right]=Q_{\pi}\left[T_{\mathrm{R}}\right]$ for any $(2,2)$-tangle $T$.

Proof. For each $T$ such that $T_{\mathrm{L}} \sim T_{\mathrm{R}}$, (5.5) yields

$$
\sum_{a}\left(\xi_{\mathrm{L} a}-\xi_{\mathrm{R} a}\right) \tau_{a}(T)=0
$$

Since there are more than $n(\Lambda) T$ 's for which the equation hold, and since $\left(\xi_{\mathrm{L} a}-\xi_{\mathrm{R} a}\right)$ is independent of $T$, (5.7) implies that $\xi_{\mathrm{L} a}-\xi_{\mathrm{R} a}=0$ for all $a$ 's. The equality $Q_{\pi}\left[T_{\mathrm{L}}\right]=Q_{\pi}\left[T_{\mathrm{R}}\right]$ then follows.

Remark. Since the number of non-isotopic $T$ 's is infinite and $n(\Lambda)$ is a finite number, without the help of a hidden symmetry, it is difficult see how a non-trivial $\pi$ could generate a trivial $\mathcal{V}_{\pi}$. For instance, the $\mathcal{V}_{\pi}$ 's corresponding to the smallest irreducible representations of the simplest quantum algebras, the fundamental, two-dimension representations of $\mathcal{U}_{q}(s l(2))$ and of $\mathcal{U}_{q}(s l(1 \mid 1))$, respectively, are already non-trivial; their $Q_{\pi}$ 's give the Jones polynomial (Jones 1985) and the Alexander-Conway polynomial (Alexander 1922, Conway 1970) (see section 7.1), respectively. 
Proof of corollary 5.2. If $T$ and $T^{\prime}$ are two $(1,1)$-tangles whose closures are isotopic to $[L]$, then it is possible to go from $\hat{T}$ to $\hat{T}^{\prime}$ via a sequence of Reidemeister moves. This implies that there exists a sequence of $(2,2)$-tangles $T_{1}, T_{2}, \ldots, T_{N}$, whose closures are all isotopic to $[L]$, and a corresponding sequence of pairs of $(1,1)$-tangles, $\left\{T_{\mathrm{L} i}, T_{\mathrm{R} i}\right\}$, that are partial closures of $T_{i}, i=1, \ldots, N$, such that $\left\{T_{\mathrm{L} i}, T_{\mathrm{R} i}\right\} \cap\left\{T_{L i+1}, T_{R i+1}\right\} \neq \emptyset, i=1, \ldots, N-1$, $T \in\left\{T_{\mathrm{L} 1}, T_{\mathrm{R} 1}\right\}$ and $T^{\prime} \in\left\{T_{\mathrm{L} N}, T_{\mathrm{R} N}\right\}$. It follows from lemma 5.1 that $Q_{\pi}[T]=Q_{\pi}\left[T^{\prime}\right]$. Corollary 5.2 is thus proven.

Remark. Thus $Q_{\pi}[T]$ maps an equivalent set larger than isotopy: for $Q_{\pi}[T]=Q_{\pi}\left[T^{\prime}\right]$, it is sufficient that $[\hat{T}] \sim\left[\hat{T}^{\prime}\right]$, and not necessary that $T \sim T^{\prime}$. See the appendix for examples.

\section{Comparison with the universal link invariant of Lawrence}

The universal link invariant of Lawrence (1989) is a Markov trace valued in the image of $\mathcal{A}_{0} \otimes l$ under the map $X_{*}$ described below. In Lawrence's construction, for the closure of a braid in $\mathcal{B} \in B_{n}$, one first writes

$$
\mathcal{V}(\mathcal{B})=x_{1} \otimes x_{2} \otimes \cdots \otimes x_{n} \quad x_{i} \in \mathcal{A}
$$

where the $x_{i}$ 's are images of strings defined in section 2.4. If $\hat{\mathcal{B}}$ has $l$ closed strings, then the sequence $(1,2, \ldots, n)$ forms $l$ cycles, and to the $r$ th cycle $\left(a_{1} \ldots a_{k}\right)$ one associates

$$
z_{r}=X_{*}{ }^{(k-1)}\left(x_{a_{k}} \cdots x_{a_{1}}\right) .
$$

The map $X_{*}: \mathcal{A} \rightarrow \mathcal{A} / I$ is the composition of $X$ (or $Y$ ) with a natural projection, where the maps $X: \mathcal{A} \rightarrow \mathcal{A}$ and $Y: \mathcal{A} \rightarrow \mathcal{A}$ are defined through their inverses in (a) below, and $I$ is an equivalence relation defined by (b) and (c):

(a) $X^{-1}(x)=m^{\prime}((x \otimes 1) \mathcal{R}), Y^{-1}(x)=m\left(\mathcal{R}^{-1}(x \otimes 1)\right)$;

(b) $X^{(j)}(x y)=X^{(j)}(y x)$;

(c) $X^{(j+1)}\left(X^{-1}(x)\right)=X^{(j+1)}\left(Y^{-1}(x)\right), x, y \in \mathcal{A}, j \geqslant 0$.

In the above, $m^{\prime}$ is the opposite multiplication and $X^{(m)}$ is $X$ composed $m$ times. Lawrence's universal invariant is given by

$$
\mathcal{V}[\hat{\mathcal{B}}]_{\text {Law }}=z_{1} \otimes \cdots \otimes z_{l}
$$

Under the equivalence I,

$$
X(x)=\bar{B} x A \sim x A \bar{B}=x v \quad Y(x)=\bar{A} x \underline{B} \sim x \underline{B} \bar{A}=x v=x \lambda^{-1} v .
$$

Thus Lawrence's invariant is valued modulo factors of $\lambda$. Conversely, if the equivalence relation (c) were not imposed, then the invariant would be for regular, instead of ambient, isotopy. For completion, we give the maps $X^{\prime}$ and $Y^{\prime}$, where

$$
\begin{aligned}
& X^{\prime-1}(x)=m(\mathcal{R}(x \otimes 1)) \quad Y^{\prime-1}(x)=m^{\prime}\left((x \otimes 1) \mathcal{R}^{-1}\right) \\
& X^{\prime}(x)=A x \bar{B} \sim x \bar{B} A=x v^{-1} \quad Y^{\prime}(x)=\underline{B} x \bar{A} \sim x \lambda v^{-1} .
\end{aligned}
$$




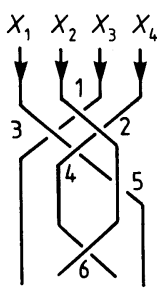

(a)

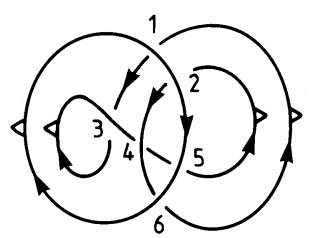

(b)

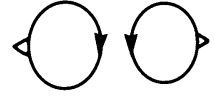

(c)

Figure 25.

For links, the actions of $X$ and $X^{\prime}$ are interchangeable, see section 2.5.6. For tangle invariants, both $X$ and $X^{\prime}$ are needed.

The difference between Lawrence's invariant and ours is illustrated in the following example. Consider the braid $\mathcal{B}$ in figure $25(a)$ and its closure $(b)$ that is isotopic to two disjoint unknots, $(c)$.

The braid has four strings whose values in $\mathcal{A}$ are, respectively,

$$
x_{1}=A_{3} B_{4} B_{5} ; \quad x_{2}=A_{1} A_{2} \bar{A}_{5} \bar{A}_{6} \quad x_{3}=B_{1} B_{3} \quad x_{4}=B_{2} \bar{A}_{4} A_{6} .
$$

The indices on $B_{1}, A_{2}$ etc label the crossings. Upon closure the indices form two cycles: $(1,4,3)$ and (2). Lawrence's invariant is

$\mathcal{V}[\hat{\mathcal{B}}]_{\text {Law }}=X_{*}^{(0)}\left(x_{2}\right) \otimes X_{*}^{(0)}\left(A A^{\prime} x_{1} x_{4} x_{3} \bar{B}^{\prime} \bar{B}\right)=X_{*}^{(0)}\left(x_{2}\right) \otimes X_{*}^{(0)}\left(A B_{4} B_{5} x_{4} B_{1} \bar{B}\right)$

which is not readily reduced to give the invariant for two disjoint unknots. However, if one uses the cyclic symmetry of $X_{*}$ after the first action of $X_{*}$ on the string $x_{1} x_{4} x_{3}$ and write $X_{*}^{(1)}\left(A^{\prime} A_{3} B_{4} B_{5} x_{4} B_{1} B_{3} \bar{B}^{\prime}\right)=X_{*}^{(1)}\left(B_{4} B_{5} x_{4} B_{1}\right)=X_{*}^{(1)}\left(x_{4} B_{1} B_{4} B_{5}\right)$, one obtains

$$
\mathcal{V}[\hat{\mathcal{B}}]_{\text {Law }}=X_{*}^{(0)}\left(x_{2}\right) \otimes X_{*}^{(0)}\left(A x_{4} B_{1} B_{4} B_{5} \bar{B}\right)
$$

which is readily reduced to give the value of two disjoint unknots. In summary, the correct way to implement the action of $X_{*}$ for the link under discussion is to write

$$
\mathcal{V}[\hat{\mathcal{B}}]_{\text {Law }}=X_{*}^{(0)}\left(x_{2}\right) \otimes X_{*}^{(0)}\left(x_{3} v^{-1} x_{1} v^{-1} x_{4}\right)=X_{*}^{(0)}(1) \otimes X_{*}^{(0)}(1) .
$$

In comparison, our method is to take the 'trace' of each cycle after it has been inserted with a factor of $v^{-1}$ or $v$ (which is equivalent for links) between each pair of adjacent strings. This gives (for figure 26(b))

$$
\mathcal{V}[\hat{\mathcal{B}}]_{\text {Lee }}=\operatorname{Sp}\left(x_{2} v^{-1}\right) \otimes S p\left(x_{3} v^{-1} x_{1} v x_{4} v\right)=\operatorname{Sp}\left(v^{-1}\right) \otimes S p(v)
$$

where the last relation makes uses of the cyclic symmetry of $S p$ and results from a series of manoeuvres corresponding to Reidemeister moves.

The following remarks are in order: 

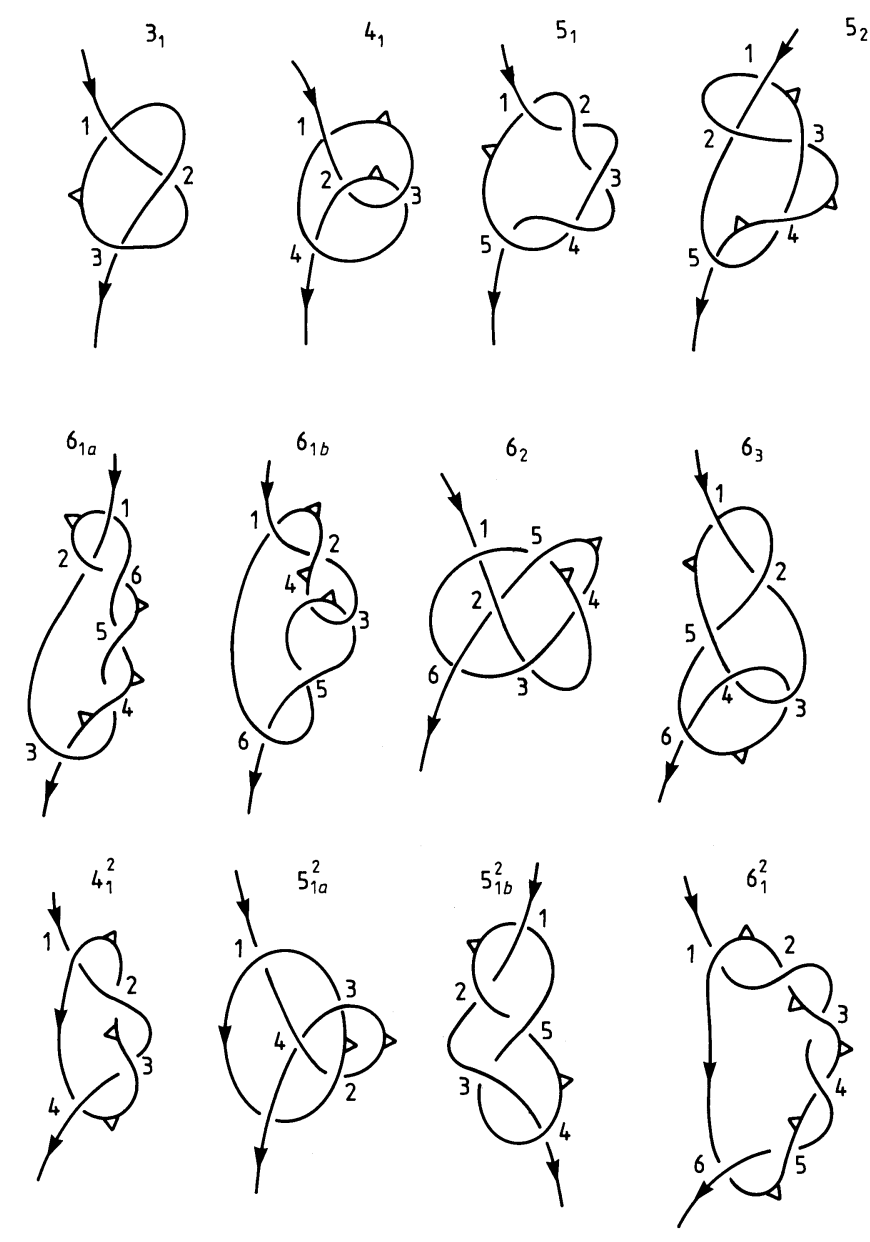

Figure 26.

(a) Lawrence's unknot is normalized to $X_{*}^{(0)}(1)$ whereas ours is normalized to $S p(v)=$ $\operatorname{Sp}\left(v^{-1}\right)$.

(b) The special role of wrong-way edges in a link is not recognized in Lawrence's construction. This is compensated by the fact that the equivalence class induced by $X_{*}$ is larger than the class given by $S p$. For instance, the two strings $x_{1} x_{4} x_{3} v^{-2}$ and $x_{3} v^{-1} x_{1} v^{-1} x_{4}$ are equivalent under $X_{*}$ but are not so under $S p$. This implies that under $X_{*}$, factors of $v^{-1}$ can be insert anywhere in the cycle, not just between two strings. This is not allowed in our construction, where each factor of $v\left(v^{-1}\right.$, resp.) is associated with a wrong-way edge on a counterclockwise (clockwise, resp.) Seifert circle.

(c) Whereas the evaluation of our invariant on a representation $\pi: \mathcal{A} \rightarrow \operatorname{End}(V)$ is straightforward-one just replaces $x \in \mathcal{A}$ by $\pi(x)$ and $S p$ by $\operatorname{Tr}_{\pi}$. The same is not true for Lawrence's invariant. In particular, $\operatorname{Tr}_{\pi}$ is not a representation of $X_{*}$; it does not have all the symmetries required of the latter. 

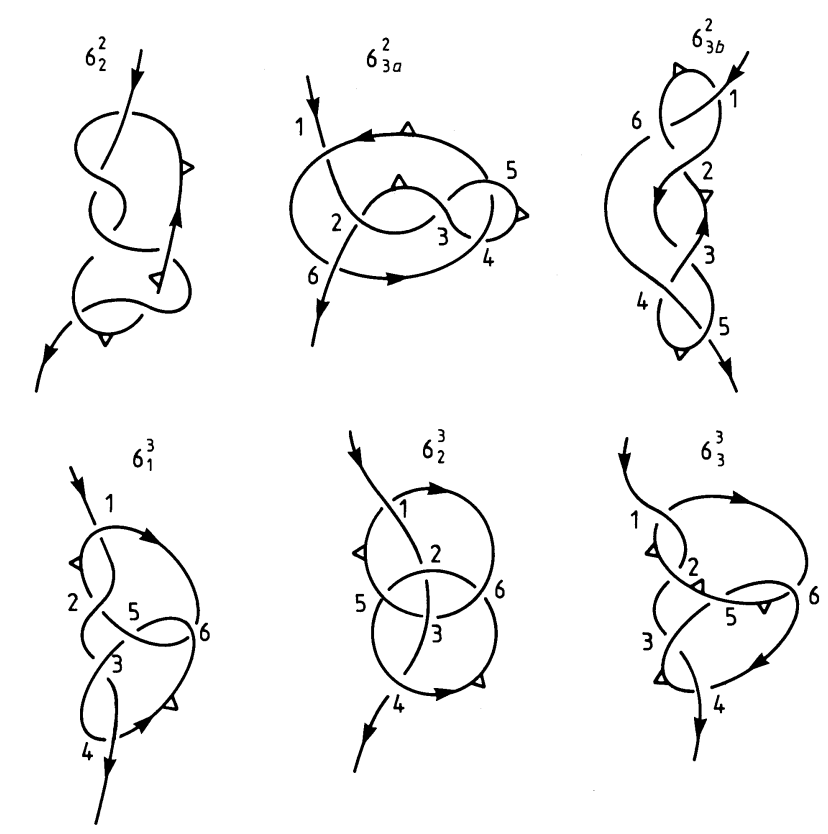

Figure 26. (Continued)

\section{Two applications}

\subsection{The Alexander-Conway polynomial}

Corollary 5.2 gives a direct proof that the Alexander-Conway polynomial (Alexander 1922, Conway 1970) is derived from an $R$-matrix having the form,

$$
\begin{aligned}
\mathcal{R}_{\pi}=s^{1 / 2}\left(s^{-1}\left(\begin{array}{cc}
1 & 0= \\
0 & 0
\end{array}\right) \otimes\left(\begin{array}{ll}
1 & 0 \\
0 & 0
\end{array}\right)-s\left(\begin{array}{ll}
0 & 0 \\
0 & 1
\end{array}\right) \otimes\left(\begin{array}{ll}
0 & 0 \\
0 & 1
\end{array}\right)+\left(\begin{array}{ll}
1 & 0 \\
0 & 0
\end{array}\right) \otimes\left(\begin{array}{ll}
0 & 0 \\
0 & 1
\end{array}\right)\right. \\
\left.+\left(\begin{array}{ll}
0 & 0 \\
0 & 1
\end{array}\right) \otimes\left(\begin{array}{ll}
1 & 0 \\
0 & 0
\end{array}\right)+\left(s^{-1}-s\right)\left(\begin{array}{ll}
0 & 0 \\
1 & 0
\end{array}\right) \otimes\left(\begin{array}{ll}
0 & 1 \\
0 & 0
\end{array}\right)\right) \\
=s^{1 / 2}\left(\begin{array}{cccc}
s^{-1} & 0 & 0 & 0 \\
0 & 1 & 0 & 0 \\
0 & s^{-1}-s & 1 & 0 \\
0 & 0 & 0 & -s
\end{array}\right)
\end{aligned}
$$

with corresponding $h_{\pi}=h_{\pi}^{-1}=s((1,0),(0,-1))$ and $\lambda_{\pi}=s^{-1}((1,0),(0,1))$. The $R$ matrix can variously be derived from braid group representations (Lee et al 1988, Couture et al 1990, Lee 1990, Deguchi 1989) and from representations of the two-parameter $\mathcal{U}_{q, s}(g l(2))$ at $q^{2}=-1$ (Lee 1989) and of $\mathcal{U}_{s}(s l(1 \mid 1)$ ) (Kauffman and Saleur 1991, Lee 1989, Zheng 1992, Links et al 1993). The $\mathcal{R}_{p} i, h_{\pi}$ and $h_{\pi}^{-1}$ have the properties needed for the functor $\mathcal{V}_{A}$ (and for $\mathcal{V}_{\pi}$ ). Now, because $\operatorname{Tr}_{\pi}\left(h_{\pi}\right)=0$, corollary 5.1 is not applicable and $\mathcal{V}_{A}[L]=0$ identically. That is, the Markov trace given by $\mathcal{R}_{\pi}$ is identically zero. This has always been a difficult point in attempts to relate the Alexander-Conway polynomial to a quantum algebra and specifically to the matrix $\mathcal{R}_{\pi}$ (Lee et al 1988, Couture et al 1990, Lee 1990, 
Kauffman and Saleur 1991, Zhang 1992, Links et al 1993). Now, with corollary 5.2, $\mathcal{V}_{A}[T]$ defines a link invariant for $\hat{T}$ through the eigenvalue $Q_{\pi}[T]$. The skein relation of this link invariant is $Q_{\pi}[\downarrow]+\left(s-s^{-1}\right) Q_{\pi}[\downarrow \downarrow]-Q_{\pi}[\precsim]=0$, it is therefore the Alexander-Conway polynomial.

Remark. Here we briefly described the relation between $\mathcal{R}_{p} i$ and the ungraded $\mathcal{U}_{q, s}(g l(2))$ at $q^{2}=-1$. Relative to $s l(2), g l(2)$ has an additional generator, call it $I$, that commutes with everything. We define a quantum algebra generated by $\left\{I, H, X^{+}, X^{-}\right\}$, where $\left\{H, X^{+}, X^{-}\right\}$ obey the rules of $\mathcal{U}_{q}(s l(2))$ with the additional properties $\Delta(I)=I \otimes 1+1 \otimes I, S(I)=-I$, $\epsilon(I)=0$. It is significant that the action of the antipode on $I$ is non-trivial. The quantum algebra $\mathcal{U}_{q}(s l(2))$ is recovered when $(s / q)^{2}=1$, a case which we ignore. Focusing on the case $(s / q)^{2} \neq 1$, one finds that there is a finite representation only if $q^{2}=\mathrm{e}^{\mathrm{i} 2 \pi / m}$, $m$ a positive integer. Furthermore, a (new) quantum algebra with the additional condition $\left(X^{ \pm}\right)^{m}=0$ can be constructed. The vector representation of the $\mathcal{R}$-matrix of this quantum algebra for $m=2$ is given by the $\mathcal{R}_{p} i$ in (7.1). Indeed, if one uses the approach of Faddeev (1988) to reconstruct a quantum formal group from $\mathcal{R}_{p} i$, then one recovers either $\mathcal{U}_{s}(s l(1 \mid 1))$ or $\mathcal{U}_{q, s}(g l(2)) /\left(\left(X^{ \pm}\right)^{2}=0\right)$ at $q^{2}=-1$, depending on whether one wants a quantum superalgebra (Zheng 1992) or a quantum algebra.

\subsection{Quantum holonomy and Chern-Simons theory in three dimensions}

Witten (1989) showed that, when the coupling constant of the Chern-Simons theory (CST) in three dimensions has certain discrete values, the Wilson line associated with the link $[L]$ evaluated in the $\pi$ representation of the gauge group $G$ is an invariant for $[L]$. A Wilson line is the character-valued counterpart of the group-valued quantum holonomy,

$$
\Phi\left(C_{x}\right)=\left\langle P \exp \left(\mathrm{i} \oint_{C_{x}} A \mathrm{~d} x\right)\right\rangle
$$

which is the expectation value of the path-ordered line integral of the connection $A(x)$ along the closed contour $C_{x}$ with initial point $x \in C$, exponentiated. In three dimensions, the contour $C$ may have non-trivial topological properties. In particular, it may be a disjoint union of contours with an opening at $x$, namely a $(1,1)$-tangle whose closure is the link $L$. Denote this tangle by $L_{x}$. Valued in the $\pi$ representation of $G$, the Wilson line with contour $L$ is

$$
W_{\pi}[L]=\operatorname{Tr}_{\pi}\left(\pi\left(\Phi\left(L_{x}\right)\right)\right)
$$

where trace taking automatically eliminates the dependence on the initial point. $W_{\pi}[L]$ is essentially the link polynomial $\mathcal{V}_{A_{\pi}}[L]$ of section 3.1 valued on the $\pi$ representation of $\mathcal{U}_{q}(g)$, where $g$ is the Lie algebra of the (Lie) group $G$, and $q$, linearly related to the coupling constant of the three-dimensional CST, is restricted to being a root of unity, $\exp (\mathrm{i} 2 \pi /(k+\tilde{h}))$, where $\tilde{h}$ is the Coxeter number of $G$ and $k$ is the level of the representation of the affine algebra associated with $g$ (Witten 1989, Horne 1990).

Recently, it was shown that for any quantum gauge theory, the quantum holonomy $\Phi\left(L_{x}\right)$ is initial-point independent and $G$-invariant (Lee and Zhu 1991). Hence for irreducible $\pi, \pi\left(\Phi\left(L_{x}\right)\right)$ must equal to an $x$-independent eigenvalue $P_{\pi}(L)$ times $\mathbf{1}_{\pi}$. In three dimensions, $L_{x}$ defines a $(1,1)$-tangle, because $L_{x}$ and $L_{y}$ may not be isotopic if $x \neq y$. 
Therefore, for the three-dimensional CST, $P_{\pi}(L)=P_{\pi}[L]$ is a link invariant. That is, the triplet $\left\{\Phi\left[L_{x}\right], P_{\pi}[L], W_{\pi}[L]\right\}$ to three-dimensional CST is what $\left\{\mathcal{V}\left[L_{x}\right], Q_{\pi}[L], \mathcal{V}_{A_{\pi}}[L]\right\}$ of section 3.3 and section 4.5 is to a quantum algebra. Thus, for example, for the gauge group $G=S L(2), P_{\pi}$ (in the fundamental representation) is the Jones polynomial (Witten 1989) and $W_{\pi}=\left(q^{-1}+q\right) P_{\pi}$; for $G=S L(M \mid N), M \neq N, P_{\pi}$ is the HOMFLY polynomial (Freyd et al 1985, Horne 1990) and $W_{\pi}=(M-N) P_{\pi}$; for $G=S L(M \mid M), P_{\pi}$ is the Alexander-Conway polynomial (Lee 1989, Lee and Zhu 1991) but $W_{\pi} \equiv 0$. That is, in quantum field theory the Alexander-Conway polynomial cannot be obtained from a Wilson line as in Witten's original approach; it must be obtained as the eigenvalue of a quantum holonomy.

\section{Acknowledgments}

The author wishes to thank J Birman for an early communication on the the importance of wrong-way edges in link invariants, V Jones for encouragement and useful information on ribbon graphs, R B Zhang for clarification on certain properties of quantum groups and Ruth Lawrence for reading the manuscript carefully and making a number of constructive suggestions for improvement. This work was partly supported by the National Science Council (ROC) under contracts 83-0208-M005-035 and 84-2112-M005-010 and by an NSERC (Canada) research grant.

\section{Appendix. Universal invariants for $(1,1)$-tangles with up to six crossings}

We list here the values of $\mathcal{V}_{A}[T]$ for $(1,1)$-tangles with up to six crossings. The tangles, shown in figure 26, are labelled by their closures using Rolfsen's classification for links (Rolfsen 1976). The wrong-way edges on each tangle are marked. The assignment of such edges is not unique but the value of the invariant of course does not vary with different assignments; see Lee (1992) for details. The indices label crossings in the tangles. The notation is as follows. $\mathcal{R}=\sum_{a} A_{a} \otimes B_{a} \equiv A \otimes B ; \mathcal{R}^{-1}=S(A) \otimes B \equiv \bar{A} \otimes B ;$ $\lambda=(m(\operatorname{id} \otimes S) \mathcal{R})\left(m^{\prime}(\operatorname{id} \otimes S) \mathcal{R}\right) ; v=\lambda^{-1 / 2}(m(\operatorname{id} \otimes S) \mathcal{R})$. Note that Rolfsen's classification is for non-oriented links and that there may be more than one non-isotopic $(1,1)$-tangles that close to the same link. In figure 26 only one orientation and one tangle is selected for each link, except for the links $6_{1}, 5_{1}^{2}$ and $6_{3}^{2}$, each for which two tangles are selected.

$$
\begin{aligned}
& 3_{1}: \lambda^{-3 / 2} \bar{A}_{1} B_{2} \bar{A}_{3} v^{-1} B_{1} \bar{A}_{2} B_{3} . \\
& 4_{1}: A_{1} B_{2} \bar{A}_{3} v B_{1} A_{4} B_{3} v \bar{A}_{2} B_{4} . \\
& 5_{1}: \lambda^{-5 / 2} \bar{A}_{1} B_{2} \bar{A}_{3} B_{4} \bar{A}_{5} v^{-1} B_{1} \bar{A}_{2} B_{3} \bar{A}_{4} B_{5} . \\
& 5_{2}: \lambda^{5 / 2} A_{1} B_{2} A_{3} B_{4} A_{5} v B_{1} A_{2} B_{5} v^{-1} A_{4} v B_{3} . \\
& 6_{1 a}: \lambda^{2} \bar{A}_{1} B_{2} A_{3} B_{4} A_{5} v B_{6} \bar{A}_{2} v^{-1} B_{1} A_{6} B_{5} v^{-1} A_{4} v^{-1} B_{3} . \\
& 6_{1 b}: \lambda^{2} A_{1} B_{2} \bar{A}_{3} B_{4} v^{-1} A_{2} v B_{1} A_{6} v B_{5} \bar{A} 4 v^{-1} B_{3} A_{5} B_{6} . \\
& 6_{2}: \lambda^{2} B_{1} A_{2} B_{3} A_{4} v B_{5} \bar{A}_{1} B_{6} A_{3} B_{4} v A_{5} B_{2} \bar{A}_{6} .
\end{aligned}
$$




$$
\begin{aligned}
& 6_{3}: \bar{A}_{1} B_{2} A_{3} B_{4} \bar{A}_{5} v^{-1} B_{1} \bar{A}_{2} B_{5} A_{6} v B_{3} A_{4} B_{6} . \\
& 4_{1}^{2}: \lambda^{-1} B_{1} \bar{A}_{2} B_{3} \bar{A}_{4} \otimes S p\left(\lambda^{-1} \bar{A}_{1} B_{4} v \bar{A}_{3} v^{-1} A_{2} v\right) . \\
& 5_{1 a}^{2}: \lambda^{1 / 2} B_{1} A_{4} B_{2} v A_{3} B_{4} \bar{A}_{5} \otimes S p\left(\bar{A}_{1} B_{5} A_{2} v B_{3}\right) . \\
& 5_{1 b}^{2}: \bar{A}_{1} B_{2} A_{3} B_{4} \otimes S p\left(\lambda^{1 / 2} B_{1} A_{5} B_{3} A_{4} v B_{5} \bar{A}_{2} v^{-1}\right) . \\
& 6_{1}^{2}: \lambda^{-3 / 2} B_{1} \bar{A}_{2} B_{3} \bar{A}_{4} B_{5} \bar{A}_{6} \otimes S p\left(\lambda^{-3 / 2} \bar{A}_{1} B_{6} v \bar{A}_{5} v^{-1} B_{4} v \bar{A}_{3} v^{-1} B_{2} v\right) . \\
& 6_{2}^{2}: \lambda^{3 / 2} A_{1} B_{2} A_{3} B_{4} A_{5} B_{6} \otimes S p\left(\lambda^{3 / 2} B_{1} A_{2} B_{3} A_{6} v B_{5} v^{-1} A_{4} v\right) . \\
& 6_{3 a}^{2}: B_{1} A_{2} B_{3} \bar{A}_{5} v^{-1} B_{4} A_{3} v B_{2} \bar{A}_{6} \otimes S p\left(\lambda^{-1} \bar{A}_{1} B_{6} \bar{A}_{4} B_{5} v\right) . \\
& 6_{3 b}^{2}: \lambda^{-1} \bar{A}_{1} B_{6} \bar{A}_{4} B_{5} \otimes S p\left(B_{1} A_{2} B_{3} \bar{A}_{5} v^{-1} B_{4} A_{3} v B_{2} \bar{A}_{6} v^{-1}\right) . \\
& 6_{1}^{3}: B_{1} A_{2} B_{3} \bar{A}_{4} \otimes S p\left(A_{1} B_{6} \bar{A}_{5} B_{2} v^{-1}\right) \otimes S p\left(\lambda^{-1} \bar{A}_{3} B_{4} v A_{6} B_{5}\right) . \\
& 6_{2}^{3}: \bar{A}_{1} B_{2} A_{3} B_{4} \otimes S p\left(B_{1} A_{6} B_{3} \bar{A}_{5} v^{-1}\right) \otimes S p\left(\bar{A}_{2} B_{5} A_{4} v B_{6}\right) . \\
& 6_{3}^{3}: \bar{A}_{1} B_{2} B_{3} A_{4} \otimes S p\left(B_{1} B_{6} v^{-1} A_{5} v \bar{A}_{2} v^{-1}\right) \otimes S p\left(\lambda A_{3} v^{-1} B_{5} A_{6} B_{4}\right) .
\end{aligned}
$$

\section{References}

Alexander J W 1922 A lemma on a system of knotted curves Proc. Natl Acad. Sci. USA 9 93-5 1928 Topological invariants of knots and links Trans. Am. Math. Soc. 30 275-306

Conway J H 1970 Computational Problems in Abstract Algebra (New York: Pergamon) p 329

Couture M, Lee H C and Schmeing N 1990 A new family of $N$-state representations of the braid group NATO ASI Ser. B 238 (New York: Plenum) pp 573-82

Drinfel'd V G 1985 Hopf algebras and the quantum Yang-Baxter equation Soviet Math. Dokl. 32 254-8

1986 Quantum groups Proc. Int. Cong. Math., Berkeley vol 1 (New York: Academic) pp 798-820

1989 On almost cocommutative Hopf algebras Alg. i Anal. $130-46$

Deguchi T 1989 Braid group representations and link polynomials derived from generalized $S U(n)$ vertex models J. Phys. Soc. Japan 58 3441-4

Faddeev L D, Reshetikhin N Yu and Takhtajan L A 1988 Quantization of Lie groups and Lie algebras Algebraic Analysis 1 129-39

Freyd P J and Yetter D N 1989 Braided compact closed categories with applications to low dimensional topology Adv. Math. 77 156-82

Freyd P, Yetter D, Hoste J, Lickorish W, Millet K and Ocneanu A 1985 Bull. Am. Math. Soc. 12 239-46

Horne J H 1990 Skein relations and Wilson loops in Chern-Simons gauge theories Nucl. Phys. B 334 669-94; equation (5.8) of this reference should read $\alpha=q^{-(m-n) / 2}, \beta=q^{1 / 2}-q^{-1 / 2}, \gamma=-q^{(m-n) / 2}$

Jimbo M 1985 A q-difference analogue of $\mathcal{U}(\mathcal{G})$ and the Yang-Baxter equation Lett. Math. Phys. 10 63-9

Jones V F R 1985 A polynomial invariant of knots via von Neumann algebras Bull. Am. Soc. 12 103-11

Kauffman L and Saleur H 1991 Free fermions and the Alexander-Conway polynomial Commun. Math. Phys 141 293-327

Lawrence Ruth 1989 A universal link invariant using quantum groups Proc. XVII Int. Conf. on Diff. Geom. Meth. Theor. Phys. (Singapore: World Scientific)

Lee H C $1989 \mathcal{U}_{q, s}(g l(2))$ at roots of unity and a hierarchy of Alexander-Conway polynomials, in preparation

1990 Q-deformation of $s l(2, C) \times Z_{N}$ and link invariants NATO ASI Ser. B 245 (New York: Plenum) pp 359-72 1992 On Seifert circles and functor for tangles Int. J. Mod. Phys. A 7, suppl 1B 581-610

Lee H C and Zhu Z Y 1991 Quantum holonomy and link invariants Phys. Rev. D 44 942-5

Lee H C, Couture M and Schmeing N 1988 Connected link polynomials Preprint (Chalk River)

Links J R, Gould M D and Zhang R B 1993 Quantum supergroups, link polynomials and representations of braid generators Rev. Math. Phys. 5 345-61

Markov A A 1935 Ueber die freie Aquivalenz geschlossener Zoepfe Recueil Math. Moscou 1 73-8 
Reidemeister K 1948 Knotentheorie (Chelsea)

Reshetikhin N Yu 1990 Quasitriangular Hopf algebras and invariants of tangles Leningrad Math. J. 1 491-513

Reshetikhin N and Turaev V G 1990 Ribbon graphs and their invariants derived from quantum groups Commun. Math. Phys. 127 1-26

Reshetikhin N Yu, Takhtadzhyan L A and Faddeev L D 1990 Quantization of Lie groups and Lie algebras Leningrad Math. J. 1 193-225

Rolfsen D 1976 Knots and links Math. Lec. Series 7 Berkeley, CA

Rozansky L and Saleur H 1992 Quantum field theory for the multi-variable Alexander-Conway polynomial Nucl. Phys. B 376 461-509

Turaev V G 1990 Operator invariants of tangles, and R-matrices Math. USSR Izv. 35 411-44

Witten E 1989 Quantum field theory and the Jones Polynomial Commun. Math. Phys. 121 351-99

Yetter D N 1988 Markov algebras Braids AMS Contemporary Mathemathics vol 78 (Providence, RI: AMS) pp 70530

Zhang R B 1992 Braid group representations arising from quantum supergroups with arbitrary $q$ and link polynomials J. Math. Phys. 33 3918-30 